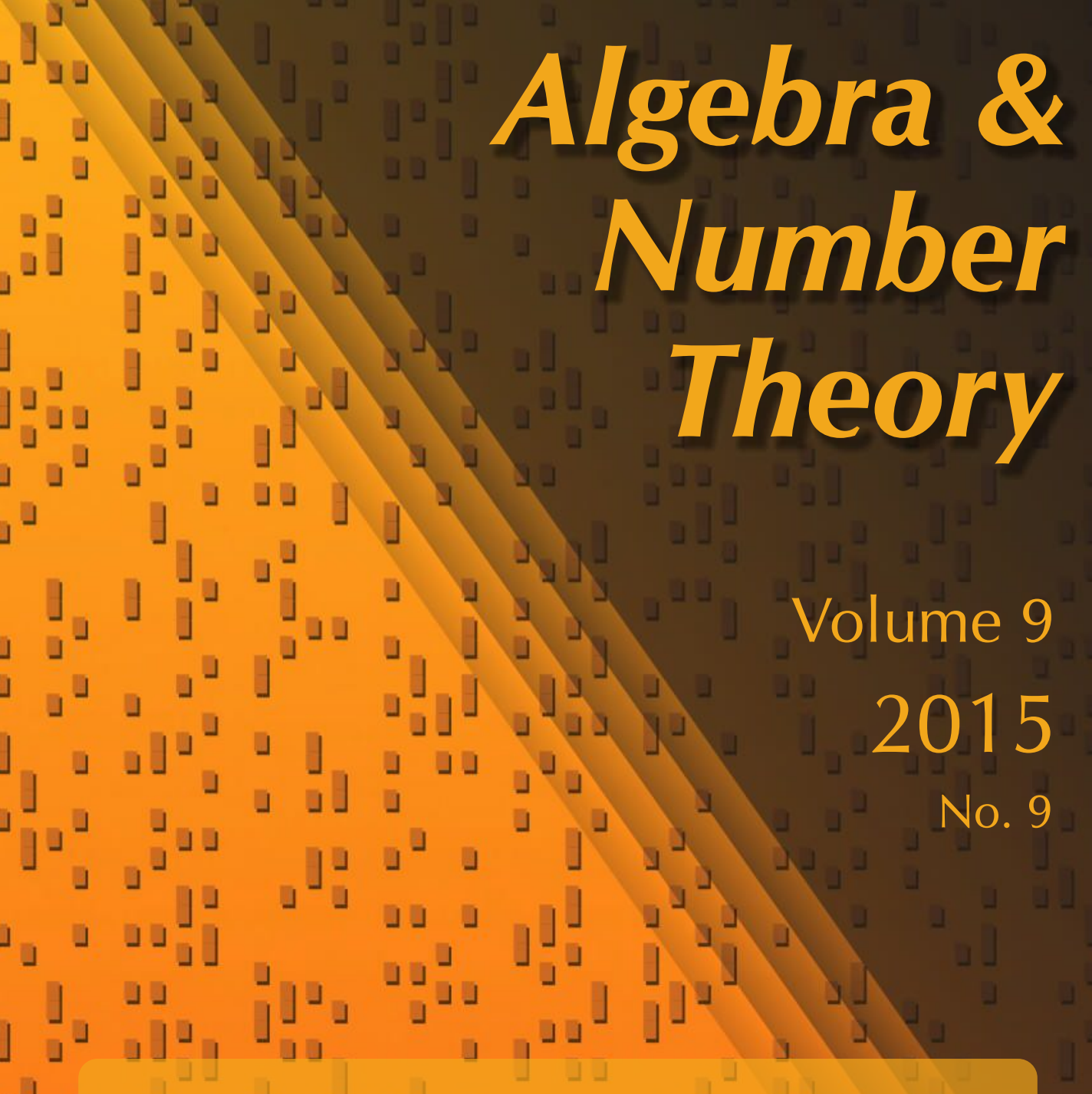




\title{
An averaged form of Chowla's conjecture
}

\author{
Kaisa Matomäki, Maksym Radziwiłł and Terence Tao
}

Let $\lambda$ denote the Liouville function. A well-known conjecture of Chowla asserts that, for any distinct natural numbers $h_{1}, \ldots, h_{k}$, one has

$$
\sum_{1 \leq n \leq X} \lambda\left(n+h_{1}\right) \cdots \lambda\left(n+h_{k}\right)=o(X)
$$

as $X \rightarrow \infty$. This conjecture remains unproven for any $h_{1}, \ldots, h_{k}$ with $k \geq 2$. Using the recent results of Matomäki and Radziwiłł on mean values of multiplicative functions in short intervals, combined with an argument of Kátai and Bourgain, Sarnak, and Ziegler, we establish an averaged version of this conjecture, namely

$$
\sum_{h_{1}, \ldots, h_{k} \leq H}\left|\sum_{1 \leq n \leq X} \lambda\left(n+h_{1}\right) \cdots \lambda\left(n+h_{k}\right)\right|=o\left(H^{k} X\right)
$$

as $X \rightarrow \infty$, whenever $H=H(X) \leq X$ goes to infinity as $X \rightarrow \infty$ and $k$ is fixed. Related to this, we give the exponential sum estimate

$$
\int_{0}^{X}\left|\sum_{x \leq n \leq x+H} \lambda(n) e(\alpha n)\right| d x=o(H X)
$$

as $X \rightarrow \infty$ uniformly for all $\alpha \in \mathbb{R}$, with $H$ as before. Our arguments in fact give quantitative bounds on the decay rate (roughly on the order of $\log \log H / \log H$ ) and extend to more general bounded multiplicative functions than the Liouville function, yielding an averaged form of a (corrected) conjecture of Elliott.

\section{Introduction}

Let $\lambda: \mathbb{N} \rightarrow\{-1,+1\}$ be the Liouville function, that is to say, the completely multiplicative function such that $\lambda(p)=-1$ for all primes $p$. The prime number theorem implies that ${ }^{1}$

$$
\sum_{1 \leq n \leq X} \lambda(n)=o(X)
$$

MSC2010: 11P32.

Keywords: multiplicative functions, Hardy-Littlewood circle method, Chowla conjecture.

${ }^{1}$ See page 2174 for our asymptotic notation conventions. 
as $X \rightarrow \infty$. More generally, a famous conjecture of Chowla [1965] asserts that, for any distinct natural numbers $h_{1}, \ldots, h_{k}$, one has

$$
\sum_{1 \leq n \leq X} \lambda\left(n+h_{1}\right) \cdots \lambda\left(n+h_{k}\right)=o(X)
$$

as $X \rightarrow \infty$.

Chowla's conjecture remains open for any $h_{1}, \ldots, h_{k}$ with $k \geq 2$. Our first main theorem establishes an averaged form of this conjecture:

Theorem 1.1 (Chowla's conjecture on average). For any natural number $k$, and any $10 \leq H \leq X$, we have

$$
\begin{aligned}
\sum_{1 \leq h_{1}, \ldots, h_{k} \leq H} \mid \sum_{1 \leq n \leq X} \lambda\left(n+h_{1}\right) \cdots \lambda(n & \left.+h_{k}\right) \mid \\
& \ll k\left(\frac{\log \log H}{\log H}+\frac{1}{\log ^{1 / 3000} X}\right) H^{k} X .
\end{aligned}
$$

In fact, we have the slightly stronger bound

$$
\begin{aligned}
\sum_{1 \leq h_{2}, \ldots, h_{k} \leq H} \mid \sum_{1 \leq n \leq X} \lambda(n) \lambda\left(n+h_{2}\right) & \cdots \lambda\left(n+h_{k}\right) \mid \\
& \ll k\left(\frac{\log \log H}{\log H}+\frac{1}{\log ^{1 / 3000} X}\right) H^{k-1} X .
\end{aligned}
$$

In the case $k=2$ our result implies that

$$
\sum_{1 \leq h \leq H}\left|\sum_{1 \leq n \leq X} \lambda(n) \lambda(n+h)\right|=o(H X)
$$

provided that $H \rightarrow \infty$ arbitrarily slowly with $X \rightarrow \infty$ (and $H \leq X$ ). Note that the $k=2$ case of Chowla's conjecture is equivalent to the above asymptotic holding in the case that $H$ is bounded rather than going to infinity.

In fact, we have a more precise bound than (1-2) (or (1-3)) that gives more control on the exceptional tuples $\left(h_{1}, \ldots, h_{k}\right)$ for which the sums of the form $\sum_{1 \leq n \leq X} \lambda\left(n+h_{1}\right) \cdots \lambda\left(n+h_{k}\right)$ are large; see Remark 5.2. In particular, in the special case $k=2$ we get the following result.

Theorem 1.2. Let $\delta \in(0,1]$ be fixed. There is a large but fixed $H=H(\delta)$ such that, for all large enough $X$,

$$
\left|\sum_{1 \leq n \leq X} \lambda(n) \lambda(n+h)\right| \leq \delta X
$$

for all but at most $H^{1-\delta / 5000}$ integers $|h| \leq H$. 
One can also replace the ranges $1 \leq h_{j} \leq H$ in Theorem 1.1 by $b_{j}+1 \leq h_{j} \leq b_{j}+H$ for any $b_{j}=O(X)$; see Theorem 1.6.

The exponents $1 / 3000$ and $1 / 5000$ in the above theorems may certainly be improved, but we did not attempt to optimize the constants here. However, our methods cannot produce a gain much larger than $1 / \log H$, as one would then have to somehow control $\lambda$ on numbers that are not divisible by any prime less than $H$, at which point we are no longer able to exploit the averaging in the $h_{1}, \ldots, h_{k}$ parameters. It would be of particular interest to obtain a gain of more than $1 / \log X$, as one could then potentially localize $\lambda$ to primes and obtain some version of the prime tuples conjecture when the $h_{1}, \ldots, h_{k}$ parameters are averaged over short intervals, but this is well beyond the capability of our methods. (If instead one is allowed to average the $h_{1}, \ldots, h_{k}$ over long intervals of scale comparable to $X$, one can obtain various averaged forms of the prime tuples conjecture and its relatives, by rather different methods than those used here; see [Balog 1990; Mikawa 1992; Kawada 1993; 1995; Green and Tao 2010].)

Theorem 1.1 is closely related to the following averaged short exponential sum estimate, which may be of independent interest.

Theorem 1.3 (exponential sum estimate). For any $10 \leq H \leq X$, one has

$$
\sup _{\alpha \in \mathbb{R}} \int_{0}^{X}\left|\sum_{x \leq n \leq x+H} \lambda(n) e(\alpha n)\right| d x \ll\left(\frac{\log \log H}{\log H}+\frac{1}{\log ^{1 / 700} X}\right) H X .
$$

Actually, for technical reasons it is convenient to prove a sharper version of Theorem 1.3 in which the Liouville function has been restricted to those numbers that have "typical" factorization; see Theorem 2.3. This sharper version will then be used to establish Theorem 1.1.

The relationship between Theorems 1.1 and 1.3 stems from the following Fourieranalytic identity:

Lemma 1.4 (Fourier identity). For $H>0$, if $f: \mathbb{Z} \rightarrow \mathbb{C}$ is a function supported on a finite set, then

$$
\int_{\mathbb{T}}\left(\int_{\mathbb{R}}\left|\sum_{x \leq n \leq x+H} f(n) e(\alpha n)\right|^{2} d x\right)^{2} d \alpha=\sum_{|h| \leq H}(H-|h|)^{2}\left|\sum_{n} f(n) \bar{f}(n+h)\right|^{2} .
$$

Proof. Using the Fourier identity $\int_{\mathbb{T}} e(n \alpha) d \alpha=1_{n=0}$, we can expand the left-hand side as

$$
\begin{aligned}
\sum_{n, n^{\prime}, m, m^{\prime}} f(n) \bar{f}\left(n^{\prime}\right) f(m) \bar{f}\left(m^{\prime}\right) 1_{n+m-n^{\prime}-m^{\prime}=0} & \\
& \times\left(\int_{\mathbb{R}} 1_{x \leq n, n^{\prime} \leq x+H} d x\right)\left(\int_{\mathbb{R}} 1_{y \leq m, m^{\prime} \leq y+H} d y\right) .
\end{aligned}
$$


Writing $n^{\prime}=n+h$, we see that both integrals are equal to $H-|h|$ if $|h| \leq H$ and vanish otherwise. The claim follows.

Theorem 1.3 may be compared with the classical estimate

$$
\sup _{\alpha \in \mathbb{R}}\left|\sum_{1 \leq n \leq X} \lambda(n) e(\alpha n)\right| \ll_{A} X \log ^{-A} X
$$

of Davenport [1937], valid for any $A>0$. Indeed, one can view Theorem 1.3 as asserting that a weak form of Davenport's estimate holds on average in short intervals. It would be of interest to also obtain nontrivial bounds on the larger quantity

$$
\int_{0}^{X} \sup _{\alpha \in \mathbb{R}}\left|\sum_{x \leq n \leq x+H} \lambda(n) e(\alpha n)\right| d x
$$

but this appears difficult to establish with our methods.

As with other applications of the circle method, our proof of Theorem 1.3 splits into two cases, depending on whether the quantity $\alpha$ is on "major arc" or on "minor arc". In the "major arc" case we are able to use the recent results of Matomäki and Radziwiłł [2015] on the average size of mean values of multiplicative functions on short intervals. Actually, in order to handle the presence of complex Dirichlet characters, we need to extend the results in [Matomäki and Radziwiłl 2015] to complex-valued multiplicative functions rather than real-valued ones; this is accomplished in an appendix to this paper (Appendix A). In the "minor arc" case we use a variant of the arguments of Kátai [1986] and Bourgain, Sarnak, and Ziegler [Bourgain et al. 2013] (see also the earlier works of Montgomery and Vaughan [1977] and Daboussi and Delange [1982]) to obtain the required cancellation. One innovation here is to rely on a combinatorial identity of Ramaré (also used in [Matomäki and Radziwiłł 2015]) as a substitute for the Turan-Kubilius inequality, as this leads to superior quantitative estimates (particularly if one first restricts the variable $n$ to have a "typical" prime factorization).

Extension to more general multiplicative functions. Define a 1-bounded multiplicative function to be a multiplicative function $f: \mathbb{N} \rightarrow \mathbb{C}$ such that $|f(n)| \leq 1$ for all $n \in \mathbb{N}$. Given two 1-bounded multiplicative functions $f, g$ and a parameter $X \geq 1$, we define the distance $\mathbb{D}(f, g ; X) \in[0,+\infty)$ by the formula

$$
\mathbb{D}(f, g ; X):=\left(\sum_{p \leq X} \frac{1-\operatorname{Re}(f(p) \overline{g(p)})}{p}\right)^{1 / 2} .
$$

This is known to give a (pseudo)metric on 1-bounded multiplicative functions; see [Granville and Soundararajan 2007, Lemma 3.1]. We also define the asymptotic 
counterpart $\mathbb{D}(f, g ; \infty) \in[0,+\infty]$ by the formula

$$
\mathbb{D}(f, g ; \infty):=\left(\sum_{p} \frac{1-\operatorname{Re}(f(p) \overline{g(p)})}{p}\right)^{1 / 2} .
$$

We informally say that $f$ pretends to be $g$ if $\mathbb{D}(f, g ; X)$ (or $\mathbb{D}(f, g ; \infty))$ is small (or finite).

For any 1-bounded multiplicative function $g$ and real number $X>1$, we introduce the quantity

$$
M(g ; X):=\inf _{|t| \leq X} \mathbb{D}\left(g, n \mapsto n^{i t} ; X\right)^{2},
$$

and then the more general quantity

$$
M(g ; X, Q):=\inf _{q \leq Q ; \chi(q)} M(g \bar{\chi} ; X)=\inf _{|t| \leq X ; q \leq Q ; \chi(q)} \mathbb{D}\left(g, n \mapsto \chi(n) n^{i t} ; X\right)^{2},
$$

where $\chi$ ranges over all Dirichlet characters of modulus $q \leq Q$. Informally, $M(g ; X)$ is small when $g$ pretends to be like a multiplicative character $n \mapsto n^{i t}$, and $M(g ; X, Q)$ is small when $g$ pretends to be like a twisted Dirichlet character of modulus at most $Q$ and twist of height at most $X$. We also define the asymptotic counterpart

$$
M(g ; \infty, \infty)=\inf _{\chi, t} \mathbb{D}\left(g, n \mapsto \chi(n) n^{i t} ; \infty\right)^{2}
$$

where $\chi$ now ranges over all Dirichlet characters and $t$ ranges over all real numbers.

Elliott proposed in [1992, Conjecture II] the following more general form of Chowla's conjecture, which we phrase here in contrapositive form.

Conjecture 1.5 (Elliott's conjecture). Let $g_{1}, \ldots, g_{k}: \mathbb{N} \rightarrow \mathbb{C}$ be 1-bounded multiplicative functions, and let $a_{1}, \ldots, a_{k}, b_{1}, \ldots, b_{k}$ be natural numbers such that any two of the pairs $\left(a_{1}, b_{1}\right), \ldots,\left(a_{k}, b_{k}\right)$ are linearly independent in $\mathbb{Q}^{2}$. Suppose that there is an index $1 \leq j_{0} \leq k$ such that

$$
M\left(g_{j_{0}} ; \infty, \infty\right)=\infty
$$

Then

$$
\sum_{1 \leq n \leq X} \prod_{j=1}^{k} g_{j}\left(a_{j} n+b_{j}\right)=o(X)
$$

as $X \rightarrow \infty$.

Informally, this conjecture asserts that for pairwise linearly independent pairs $\left(a_{1}, b_{1}\right), \ldots,\left(a_{k}, b_{k}\right)$ and any 1-bounded multiplicative $g_{1}, \ldots, g_{k}$, one has the asymptotic (1-8) as $X \rightarrow \infty$, unless each of the $g_{j}$ pretends to be a twisted Dirichlet character $n \mapsto \chi_{j}(n) n^{i t_{j}}$. Note that some condition of this form is necessary, since if $g(n)$ is equal to $\chi(n) n^{i t}$ then $g(n) \overline{g(n+h)}$ will be biased to be positive for large $n$, if $h$ is fixed and divisible by the modulus $q$ of $\chi$; one also expects some bias when 
$h$ is not divisible by this modulus since the sums $\sum_{n \in \mathbb{Z} / q \mathbb{Z}} \chi(n) \overline{\chi(n+h)}$ do not vanish in general. From the prime number theorem in arithmetic progressions it follows that

$$
M(\lambda ; \infty, \infty)=\infty
$$

so Elliott's conjecture implies Chowla's conjecture (1-1).

When one allows the functions $g_{j}$ to be complex-valued rather than real-valued, Elliott's conjecture turns out to be false on a technicality; one can choose 1-bounded multiplicative functions $g_{j}$ which are arbitrarily close at various scales to a sequence of functions of the form $n \mapsto n^{i t_{m}}$ (which allows one to violate (1-8)) without globally pretending to be $n^{i t}$ (or $\chi(n) n^{i t}$ ) for any fixed $t$; we present this counterexample in Appendix B. However, this counterexample can be removed by replacing (1-7) with the stronger condition that

$$
M\left(g_{j_{0}} ; X, Q\right) \rightarrow \infty
$$

as $X \rightarrow \infty$ for each fixed $Q$. In the real-valued case, (1-9) and (1-7) are equivalent by a triangle inequality argument of Granville and Soundararajan which we give in Appendix C.

As evidence for the corrected form of Conjecture 1.5 (in both the real-valued and complex-valued cases), we present the following averaged form of that conjecture:

Theorem 1.6 (Elliott's conjecture on average). Let $10 \leq H \leq X$ and $A \geq 1$. Let $g_{1}, \ldots, g_{k}: \mathbb{N} \rightarrow \mathbb{C}$ be 1-bounded functions, and let $a_{1}, \ldots, a_{k}, b_{1}, \ldots, b_{k}$ be natural numbers with $a_{j} \leq A$ and $b_{j} \leq A X$ for $j=1, \ldots, k$. Let $1 \leq j_{0} \leq k$, and suppose that $g_{j_{0}}$ is multiplicative. Then one has

$$
\begin{aligned}
\sum_{1 \leq h_{1}, \ldots, h_{k} \leq H}\left|\sum_{1 \leq n \leq X} \prod_{j=1}^{k} g_{j}\left(a_{j} n+b_{j}+h_{j}\right)\right| \\
\ll A^{2} k\left(\exp (-M / 80)+\frac{\log \log H}{\log H}+\frac{1}{\log ^{1 / 3000} X}\right) H^{k} X
\end{aligned}
$$

where

$$
M:=M\left(g_{j_{0}} ; 10 A X, Q\right) \quad \text { and } \quad Q:=\min \left(\log ^{1 / 125} X, \log ^{20} H\right) .
$$

In fact, we have the slightly stronger bound

$$
\begin{aligned}
\sum_{1 \leq h_{2}, \ldots, h_{k} \leq H} \mid & \sum_{1 \leq n \leq X} g_{1}\left(a_{1} n+b_{1}\right) \prod_{j=2}^{k} g_{j}\left(a_{j} n+b_{j}+h_{j}\right) \mid \\
& \ll A^{2} k\left(\exp (-M / 80)+\frac{\log \log H}{\log H}+\frac{1}{\log ^{1 / 3000} X}\right) H^{k-1} X .
\end{aligned}
$$


Note that if $a_{1}, \ldots, a_{k}, b_{1}, \ldots, b_{k}$ are fixed, $g_{j_{0}}$ is independent of $X$ and obeys the condition (1-9) for any fixed $Q$, and $H=H(X)$ is chosen to go to infinity arbitrarily slowly as $X \rightarrow \infty$, then the quantity $M$ in the above theorem goes to infinity (note that $M(g ; X, Q)$ is nondecreasing in $Q$ ), and (1-11) then implies an averaged form of the asymptotic (1-8). Thus Theorem 1.6 is indeed an averaged form of the corrected form of Conjecture 1.5. (We discovered the counterexample in Appendix B while trying to interpret Theorem 1.6 as an averaged version of the original form of Conjecture 1.5.) Interestingly, only one of the functions $g_{1}, \ldots, g_{k}$ in Theorem 1.6 is required to be multiplicative, ${ }^{2}$ one can use a van der Corput argument to reduce matters to obtaining cancellation for a sum roughly of the form $\sum_{h \leq H}\left|\sum_{1 \leq n \leq X} g_{j_{0}}(n) \overline{g_{j_{0}}(n+h)}\right|^{2}$, which can then be treated using Lemma 1.4.

For $g(n)=\lambda(n)$ and $X, Q, M$ as in the above theorem, one obtains, for every $\varepsilon>0$, the bound

$$
\begin{aligned}
M & \geq \inf _{|t| \leq X ; q \leq Q ; \chi(q)} \sum_{\exp \left((\log X)^{2 / 3+\varepsilon}\right) \leq p \leq X} \frac{1+\operatorname{Re} \chi(p) p^{i t}}{p} \\
& \geq\left(\frac{1}{3}-\varepsilon\right) \log \log X+O(1) .
\end{aligned}
$$

The last inequality is established via standard methods from the VinogradovKorobov type zero-free region

$$
\left\{\sigma+i t: \sigma>1-\frac{c}{\max \left\{\log q,(\log (3+|t|))^{2 / 3}(\log \log (3+|t|))^{1 / 3}\right\}}\right\}
$$

for $L(s, \chi)$ and some absolute constant $c>0$, which applies since $\chi$ has conductor $q \leq(\log X)^{1 / 125}$ (so that there are no exceptional zeros); see [Montgomery 1994, §9.5]. Hence Theorem 1.6 implies Theorem 1.1. The same argument gives Theorem 1.1 when the Liouville function $\lambda$ is replaced by the Möbius function $\mu$. We remark that, as our arguments make no use of exceptional zeroes, all the implied constants in our theorems are effective.

We also have a generalized form of Theorem 1.3:

Theorem 1.7 (exponential sum estimate). Let $X \geq H \geq 10$ and let $g$ be a 1-bounded multiplicative function. Then

$$
\begin{aligned}
\sup _{\alpha \in \mathbb{T}} \int_{0}^{X}\left|\sum_{x \leq n \leq x+H} g(n) e(\alpha n)\right| d x & \\
\ll & \left(\exp (-M(g ; X, Q) / 20)+\frac{\log \log H}{\log H}+\frac{1}{\log ^{1 / 700} X}\right) H X
\end{aligned}
$$

${ }^{2}$ We thank the referee for observing this fact. In a previous version of this paper, all of the $g_{j}$ were required to be multiplicative. 
where

$$
Q:=\min \left(\log ^{1 / 125} X, \log ^{5} H\right) .
$$

By (1-12), Theorem 1.7 implies Theorem 1.3.

Remark 1.8. In the recent preprint [Frantzikinakis and Host 2015], a different averaged form of Elliott's conjecture is established, in which one uses fewer averaging parameters $h_{i}$ than in Theorem 1.6 (indeed, one can average over just a single such parameter, provided that the linear parts of the forms are independent), but the averaging parameters range over a long range (comparable to $X$ ) rather than on the short range given here. The methods of proof are rather different (in particular, the arguments in [Frantzikinakis and Host 2015] rely on higher order Fourier analysis). In the long-range averaged situation considered in [Frantzikinakis and Host 2015], the counterexample in Appendix B does not apply, and one can use the original form of Elliott's conjecture in place of the corrected version. It may be possible to combine the results here with those in [Frantzikinakis and Host 2015] to obtain an averaged version of Chowla's or Elliott's conjecture in which the number of averaging parameters is small, and the averaging is over a short range, but this seems to require nontrivial estimates on quantities such as (1-5), which we are currently unable to handle.

Notation. Our asymptotic notation conventions are as follows. We use $X \ll Y$, $Y \gg X$, or $X=O(Y)$ to denote the estimate $|X| \leq C Y$ for some absolute constant $C$. If $x$ is a parameter going to infinity, we use $X=o(Y)$ to denote the claim that $|X| \leq c(x) Y$ for some quantity $c(x)$ that goes to zero as $x \rightarrow \infty$ (holding all other parameters fixed).

Unless otherwise specified, all sums are over the integers, except for sums over the variable $p$ (or $p_{1}, p_{2}$, etc.) which are understood to be over primes.

We use $\mathbb{T}:=\mathbb{R} / \mathbb{Z}$ to denote the standard unit circle and let $e: \mathbb{T} \rightarrow \mathbb{C}$ be the standard character $e(x):=e^{2 \pi i x}$.

We use $1_{S}$ to denote the indicator of a predicate $S$, thus $1_{S}=1$ when $S$ is true and $1_{S}=0$ when $S$ is false. If $A$ is a set, we write $1_{A(n)}$ for $1_{n \in A}$, so that $1_{A}$ is the indicator function of $A$.

\section{Restricting to numbers with typical factorization}

To prove Theorem 1.6 and Theorem 1.7 (and hence Theorem 1.1 and Theorem 1.3), it is technically convenient (as in the previous paper [Matomäki and Radziwiłł 2015]) to restrict the support of the multiplicative functions to a certain dense set $\mathcal{S}$ of natural numbers that have a "typical" prime factorization in a certain specific sense, in order to fully exploit a useful combinatorial identity of Ramaré (see (3-2)). 
This will lead to improved quantitative estimates in the arguments in subsequent sections of the paper.

More precisely, we introduce the following sets $\mathcal{S}$ of numbers with typical prime factorization, which previously appeared in [Matomäki and Radziwiłł 2015].

Definition 2.1. Let $10<P_{1}<Q_{1} \leq X$ and $\sqrt{X} \leq X_{0} \leq X$ be quantities such that $Q_{1} \leq \exp \left(\sqrt{\log X_{0}}\right)$. We then define $P_{j}, Q_{j}$ for $j>1$ by the formulas

$$
P_{j}:=\exp \left(j^{4 j}\left(\log Q_{1}\right)^{j-1} \log P_{1}\right), \quad Q_{j}:=\exp \left(j^{4 j+2}\left(\log Q_{1}\right)^{j}\right)
$$

for $j>1$. Note that the intervals $\left[P_{j}, Q_{j}\right]$ are disjoint and increase to infinity; indeed, one easily verifies that

$$
P_{1}<Q_{1}<\exp \left(2^{8} \log Q_{1} \log P_{1}\right)=P_{2}
$$

and

$$
P_{j}<\exp \left(j^{4 j}\left(\log Q_{1}\right)^{j}\right)<Q_{j}<\exp \left((j+1)^{4(j+1)}\left(\log Q_{1}\right)^{j}\right)<P_{j+1}
$$

for all $j>1$. Let $J$ be the largest index such that $Q_{J} \leq \exp \left(\sqrt{\log X_{0}}\right)$. Then we define $\mathcal{S}_{P_{1}, Q_{1}, X_{0}, X}$ to be the set of all the numbers $1 \leq n \leq X$ which have at least one prime factor in the interval $\left[P_{j}, Q_{j}\right]$ for each $1 \leq j \leq J$.

In practice, $X$ will be taken to be slightly smaller than $X_{0}^{2}$. The need to have two parameters $X, X_{0}$ instead of one is technical (we need to have the freedom later in the argument to replace $X$ with a slightly smaller quantity $X / d$ without altering $J$ ), but the reader may wish to pretend that $X_{0}=\sqrt{X}$ for most of the argument.

This set is fairly dense if $P_{1}$ and $Q_{1}$ are widely separated:

Lemma 2.2. Let $10<P_{1}<Q_{1} \leq X$ and $\sqrt{X} \leq X_{0} \leq X$ be quantities such that $Q_{1} \leq \exp \left(\sqrt{\log X_{0}}\right)$. Then, for every large enough $X$,

$$
\#\left\{1 \leq n \leq X: n \notin \mathcal{S}_{P_{1}, Q_{1}, X_{0}, X}\right\} \ll \frac{\log P_{1}}{\log Q_{1}} \cdot X .
$$

Proof. From the fundamental lemma of sieve theory (see, e.g., [Friedlander and Iwaniec 2010, Theorem 6.17]) we know that, for any $1 \leq j \leq J$ and large enough $X$, the number of $1 \leq n \leq X$ that are not divisible by any prime in $\left[P_{j}, Q_{j}\right]$ is at most

$$
\ll X \prod_{P_{j} \leq p \leq Q_{j}}\left(1-\frac{1}{p}\right) \ll \frac{\log P_{j}}{\log Q_{j}} X=\frac{1}{j^{2}} \frac{\log P_{1}}{\log Q_{1}} X .
$$

Summing over $j$, we obtain the claim.

Both Theorems 1.6 and 1.7 will be deduced from the following claim.

Theorem 2.3 (key exponential sum estimate). Let $X, H, W \geq 10$ be such that

$$
(\log H)^{5} \leq W \leq \min \left\{H^{1 / 250},(\log X)^{1 / 125}\right\}
$$


and let $g$ be a 1-bounded multiplicative function such that

$$
W \leq \exp (M(g ; X, Q) / 3) .
$$

Set

$$
\mathcal{S}:=\mathcal{S}_{P_{1}, Q_{1}, \sqrt{X}, X} \quad \text { where } P_{1}:=W^{200}, Q_{1}:=H / W^{3} .
$$

Then, for any $\alpha \in \mathbb{T}$, one has

$$
\int_{\mathbb{R}}\left|\sum_{x \leq n \leq x+H} 1_{\mathcal{S}}(n) g(n) e(\alpha n)\right| d x \ll \frac{(\log H)^{1 / 4} \log \log H}{W^{1 / 4}} H X .
$$

In Section 5 we will show how this theorem implies Theorem 1.6. For now, let us at least see how it implies Theorem 1.7:

Proof of Theorem 1.7 assuming Theorem 2.3. We may assume that $X, H$, and $M(g ; X, Q)$ are larger than any specified absolute constant, since if one of these expressions is bounded, then so is $W$. The claim (2-2) is then trivial with a suitable choice of implied constant (discarding the $(\log H)^{1 / 4} \log \log H$ factor).

Choose $H_{0}$ such that

$$
\log H_{0}:=\min \left(\log ^{1 / 700} X \log \log X, \exp (M(g ; X, Q) / 20) M(g ; X, Q)\right) .
$$

We divide into two cases: $H \leq H_{0}$ and $H>H_{0}$.

First suppose that $H \leq H_{0}$. Then if we set $W:=\log ^{5} H$, one verifies that all the hypotheses of Theorem 2.3 hold, and hence

$$
\int_{0}^{X}\left|\sum_{x \leq n \leq x+H} 1_{\mathcal{S}}(n) g(n) e(\alpha n)\right| d x \ll \frac{\log \log H}{\log H} H X .
$$

On the other hand, from Lemma 2.2, the choice of $W, P_{1}, Q_{1}$, and the bound on $H$, we see that

$$
\#\{1 \leq n \leq X+H: n \notin \mathcal{S}\} \ll \frac{\log \log H}{\log H} X
$$

and thus, by Fubini's theorem and the triangle inequality,

$$
\int_{0}^{X}\left|\sum_{x \leq n \leq x+H}\left(1-1_{\mathcal{S}}(n)\right) g(n) e(\alpha n)\right| d x \ll \frac{\log \log H}{\log H} H X .
$$

Summing, we obtain Theorem 1.7 in this case.

Now suppose that $H>H_{0}$. Covering $[0, H]$ by $O\left(H / H_{0}\right)$ intervals of length $H_{0}$, we see that

$$
\int_{0}^{X}\left|\sum_{x \leq n \leq x+H} g(n) e(\alpha n)\right| d x \ll \frac{H}{H_{0}} \int_{0}^{X+H}\left|\sum_{x \leq n \leq x+H_{0}} g(n) e(\alpha n)\right| d x .
$$


Also, observe from the choice of $H_{0}$ that the quantity

$$
\exp (-M(g ; X, Q) / 20)+\frac{\log \log H}{\log H}+\frac{1}{\log ^{1 / 700} X}
$$

is unchanged up to multiplicative constants if one reduces $H$ to $H_{0}$. Finally, from Mertens' theorem we see that $M(g ; X+H, Q)=M(g ; X, Q)+O(1)$. The claim then follows from the $H=H_{0}$ case (after performing the minor alteration of replacing $X$ with $X+H$ ).

We now begin the proof of Theorem 2.3. The first step is to reduce to the case where $g$ is completely multiplicative rather than multiplicative. More precisely, we will deduce Theorem 2.3 from the following proposition.

Proposition 2.4 (completely multiplicative exponential sum estimate). Assume $X, H, W \geq 10$ are such that

$$
(\log H)^{5} \leq W \leq \min \left\{H^{1 / 250},(\log X)^{1 / 125}\right\}
$$

and let $g$ be a 1-bounded completely multiplicative function such that

$$
W \leq \exp (M(g ; X, W) / 3) .
$$

Let $d$ be a natural number with $d<W$. Set

$$
\mathcal{S}:=\mathcal{S}_{P_{1}, Q_{1}, \sqrt{X}, X / d} \text { where } P_{1}:=W^{200}, Q_{1}:=H / W^{3} .
$$

Then for any $\alpha \in \mathbb{T}$ one has

$$
\left.\int_{\mathbb{R}}\right|_{x / d \leq n \leq x / d+H / d} 1_{\mathcal{S}}(n) g(n) e(\alpha n) \mid d x \ll \frac{1}{d^{3 / 4}} \frac{(\log H)^{1 / 4} \log \log H}{W^{1 / 4}} H X .
$$

Let us explain why Theorem 2.3 follows from Proposition 2.4. Let the hypotheses and notation be as in Theorem 2.3. The function $g$ is not necessarily completely multiplicative, but we may approximate it by the 1-bounded completely multiplicative function $g_{1}: \mathbb{N} \rightarrow \mathbb{C}$, defined as the completely multiplicative function with $g_{1}(p)=g(p)$ for all primes $p$. By Möbius inversion we may then write $g=g_{1} * h$ where $*$ denotes Dirichlet convolution and $h$ is the multiplicative function $h=g * \mu g_{1}$. Observe that, for all primes $p$, we have $h(p)=0$ and $\left|h\left(p^{j}\right)\right| \leq 2$ for $j \geq 2$. We now write

$$
\sum_{x \leq n \leq x+H} 1_{\mathcal{S}_{P_{1}, Q_{1}, \sqrt{X}, X}} g(n) e(\alpha n)=\sum_{d=1}^{\infty} h(d) \sum_{x / d \leq m \leq x / d+H / d} 1_{\mathcal{S}_{P_{1}, Q_{1}, \sqrt{X}, X}}(d m) g_{1}(m) e(d \alpha m)
$$


and so by the triangle inequality we may upper bound the left-hand side of (2-2) by

$$
\sum_{d=1}^{\infty}|h(d)| \int_{\mathbb{R}}\left|\sum_{x / d \leq m \leq x / d+H / d} 1_{\mathcal{S}_{P_{1}, Q_{1}, \sqrt{X}, X}}(d m) g_{1}(m) e(d \alpha m)\right| d x .
$$

Let us first dispose of the contribution where $d \geq W$. Here we trivially bound this contribution by

$$
\sum_{d \geq W}|h(d)| \sum_{m \leq(2 X+H) / d} O(H)
$$

(after moving the absolute values inside the $m$ summation and then performing the integration on $x$ first). We can bound this in turn by

$$
\ll H X \frac{1}{W^{1 / 4}} \sum_{d=1}^{\infty} \frac{|h(d)|}{d^{3 / 4}} .
$$

From Euler products we see that $\sum_{d=1}^{\infty}|h(d)| / d^{3 / 4}=O(1)$, so the contribution of this case is acceptable.

Now we consider the contribution $d<W<P_{1}$. In this case we may reduce

$$
1_{\mathcal{S}_{P_{1}, Q_{1}, \sqrt{X}, X}}(d m)=1_{\mathcal{S}_{P_{1}, Q_{1}, \sqrt{X}, X / d}}(m)
$$

and so this contribution to (2-2) can be upper bounded by

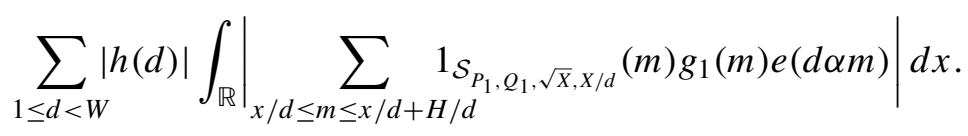

By Proposition 2.4, this is bounded by

$$
\sum_{d=1}^{\infty} \frac{|h(d)|}{d^{3 / 4}} \frac{(\log H)^{1 / 4} \log \log H}{W^{1 / 4}} H X .
$$

As before, we have $\sum_{d=1}^{\infty}|h(d)| / d^{3 / 4}=O(1)$, and Theorem 2.3 follows.

It remains to prove Proposition 2.4. For any $\alpha \in \mathbb{T}$, we know from the Dirichlet approximation theorem that there exists a rational number $a / q$ with $(a, q)=1$ and $1 \leq q \leq H / W$ such that

$$
\left|\alpha-\frac{a}{q}\right| \leq \frac{W}{q H} \leq \frac{1}{q^{2}}
$$

In the next two sections, we will apply separate arguments to prove Proposition 2.4 in the minor arc case $q>W$ and the major arc case $q \leq W$. 


\section{Proof of minor arc estimate}

We now prove Proposition 2.4 in the minor arc case $q>W$. It suffices to show that

$$
\int_{\mathbb{R}} \theta(x) \sum_{x / d \leq n \leq x / d+H / d} 1_{\mathcal{S}}(n) g(n) e(\alpha n) d x \ll \frac{1}{d^{3 / 4}} \frac{(\log H)^{1 / 4} \log \log H}{W^{1 / 4}} H X
$$

whenever $\theta: \mathbb{R} \rightarrow \mathbb{C}$ is measurable, with $|\theta(x)|$ at most 1 for all $x$ and supported on $[0, X]$. We will now use a variant of an idea of Bourgain, Sarnak, and Ziegler [Bourgain et al. 2013] (building on earlier works of Kátai [1986], Montgomery and Vaughan [1977] and Daboussi and Delange [1982]).

Let $\mathcal{P}$ be the set consisting of the primes lying between $P_{1}$ and $Q_{1}$. Then notice that each $n \in \mathcal{S}$ has at least one prime factor from $\mathcal{P}$. This leads to the following variant of Ramaré's identity (see [Friedlander and Iwaniec 2010, Section 17.3]):

$$
1_{\mathcal{S}}(n)=\sum_{p \in \mathcal{P}, m: m p=n} \frac{1_{\mathcal{S}^{\prime}}(m p)}{1+\#\{q \mid m: q \in \mathcal{P}\}},
$$

where $\mathcal{S}^{\prime}$ is the set of all $1 \leq n \leq X / d$ that have at least one prime factor in each of the intervals $\left[P_{j}, Q_{j}\right]$ for $j \geq 2$; the constraint $n \leq X / d$ arises from the corresponding constraint in the definition of $\mathcal{S}$.

Using this identity, we may write the left-hand side of (3-1) as

$$
\sum_{p \in \mathcal{P}} \sum_{m} \frac{1_{\mathcal{S}^{\prime}}(m p) g(m p) e(m p \alpha)}{1+\#\{q \mid m: q \in \mathcal{P}\}} \int_{\mathbb{R}} \theta(x) 1_{x / d \leq m p \leq(x+H) / d} d x .
$$

As $g$ is completely multiplicative, $g(m p)=g(m) g(p)$. Thus it suffices to show that $\sum_{p \in \mathcal{P}} \sum_{m} \frac{1_{\mathcal{S}^{\prime}}(m p) g(m) g(p) e(m p \alpha)}{1+\#\{q \mid m: q \in \mathcal{P}\}} \int_{\mathbb{R}} \theta(x) 1_{x / d \leq m p \leq(x+H) / d} d x$

$$
\ll \frac{(\log H)^{1 / 4} \log \log H}{d^{3 / 4} W^{1 / 4}} H X .
$$

We can cover $\mathcal{P}$ by intervals $[P, 2 P]$ with $P_{1} \ll P \ll Q_{1}$ and $P$ a power of two, and observe that

$$
\sum_{\substack{P_{1} \ll P \ll Q_{1} \\ P=2^{j}}} \frac{1}{\log P} \ll \log \log Q_{1}-\log \log P_{1} \ll \log \log H,
$$

so by the triangle inequality it suffices to show that

$$
\begin{aligned}
\sum_{\substack{p \in \mathcal{P} \\
P \leq p \leq 2 P}} \sum_{m} \frac{1_{\mathcal{S}^{\prime}}(m p) g(m) g(p) e(m p \alpha)}{1+\#\{q \mid m: q \in \mathcal{P}\}} \int_{\mathbb{R}} \theta(x) 1_{x / d \leq m p \leq(x+H) / d} d x & \\
& \ll \frac{(\log H)^{1 / 4}}{d^{3 / 4} W^{1 / 4} \log P} H X
\end{aligned}
$$


for each such $P$. We can rearrange the left-hand side as

$$
\sum_{m \in \mathcal{S}^{\prime}} \frac{g(m)}{1+\#\{q \mid m: q \in \mathcal{P}\}} \sum_{\substack{p \in \mathcal{P} \\ P \leq p \leq 2 P}} 1_{m p \leq X / d} g(p) e(m p \alpha) \int_{\mathbb{R}} \theta(x) 1_{x / d \leq m p \leq(x+H) / d} d x .
$$

Observe that the summand vanishes unless we have $m \leq X / d P$. Crudely bounding ${ }^{3}$ $g(m) /(1+\#\{q \mid m: q \in \mathcal{P}\})$ in magnitude by 1 and applying Hölder's inequality, we may bound the previous expression in magnitude by

$$
\left(\frac{X}{d P}\right)^{3 / 4}\left(\sum_{m \leq X / d P}\left|\sum_{\substack{p \in \mathcal{P} \\ P \leq p \leq 2 P}} 1_{m p \leq X / d} g(p) e(m p \alpha) \int_{\mathbb{R}} \theta(x) 1_{x / d \leq m p \leq(x+H) / d}\right|^{4} d x\right)^{1 / 4} .
$$

It thus suffices to show that

$$
\begin{aligned}
& \sum_{m \leq X / d P}\left|\sum_{\substack{p \in \mathcal{P} \\
P \leq p \leq 2 P}} 1_{m p \leq X / d} g(p) e(m p \alpha) \int_{\mathbb{R}} \theta(x) 1_{x / d \leq m p \leq(x+H) / d} d x\right|^{4} \\
& \ll \frac{\log H}{W \log ^{4} P} H^{4} X P^{3} .
\end{aligned}
$$

The left-hand side may be expanded as

$$
\begin{aligned}
\sum_{\substack{p_{1}, p_{2}, p_{3}, p_{4} \in \mathcal{P} \\
P \leq p_{1}, p_{2}, p_{3}, p_{4} \leq 2 P}} \int \cdots \int & g\left(p_{1}\right) g\left(p_{2}\right) \overline{g\left(p_{3}\right)} \overline{g\left(p_{4}\right)} \theta\left(x_{1}\right) \theta\left(x_{2}\right) \overline{\theta\left(x_{3}\right)} \overline{\theta\left(x_{4}\right)} \\
& \times \sum_{\substack{m \leq X /\left(d p_{i}\right) \\
x_{i} /\left(d p_{i}\right) \leq m \leq\left(x_{i}+H\right) /\left(d p_{i}\right) \\
\forall i=1,2,3,4}} e\left(m\left(p_{1}+p_{2}-p_{3}-p_{4}\right) \alpha\right) d x_{1} d x_{2} d x_{3} d x_{4} .
\end{aligned}
$$

From summing the geometric series, we observe that the summation over $m$ is $O\left(\min \left(H / P, 1 /\left\|\left(p_{1}+p_{2}-p_{3}-p_{4}\right) \alpha\right\|\right)\right)$, where $\|z\|$ denotes the distance from $z$ to the nearest integer. Also, the sum vanishes unless we have $x_{1}=O(X)$ and $x_{i}=x_{1} p_{i} / p_{1}+O(H)$ for $i=2,3,4$, so there are only $O\left(X H^{3}\right)$ quadruples $\left(x_{1}, x_{2}, x_{3}, x_{4}\right)$ which contribute. Thus we may bound the previous expression by

$$
O\left(X H^{3} \sum_{p_{1}, p_{2}, p_{3}, p_{4} \leq 2 P} \min \left(\frac{H}{P}, \frac{1}{\left\|\left(p_{1}+p_{2}-p_{3}-p_{4}\right) \alpha\right\|}\right)\right)
$$

and so we reduce to showing that

$$
\sum_{p_{1}, p_{2}, p_{3}, p_{4} \leq 2 P} \min \left(\frac{H}{P}, \frac{1}{\left\|\left(p_{1}+p_{2}-p_{3}-p_{4}\right) \alpha\right\|}\right) \ll \log H \frac{H P^{3}}{W \log ^{4} P} .
$$

${ }^{3}$ By using the Turan-Kubilius inequality here one could save a factor of $\log \log H$, but such a gain will not make a significant impact on our final estimates. 
The quantity $p_{1}+p_{2}-p_{3}-p_{4}$ is clearly of size $O(P)$. Conversely, from a standard upper bound sieve, ${ }^{4}$ the number of representations of an integer $n=O(P)$ of the form $p_{1}+p_{2}-p_{3}-p_{4}$ with $p_{1}, p_{2}, p_{3}, p_{4} \leq 2 P$ prime is $O\left(P^{3} / \log ^{4} P\right)$. Thus it suffices to show that

$$
\sum_{n=O(P)} \min \left(\frac{H}{P}, \frac{1}{\|n \alpha\|}\right) \ll \frac{\log H}{W} H .
$$

But from the Vinogradov lemma (see, e.g., [Iwaniec and Kowalski 2004, page 346]), the left-hand side is bounded by

$$
O\left(\left(\frac{P}{q}+1\right)\left(\frac{H}{P}+q \log q\right)\right) \ll \frac{H}{q}+P \log q+\frac{H}{P}+q \log q
$$

which, since

$$
W^{200}=P_{1} \ll P \ll Q_{1}=H / W^{3} \quad \text { and } \quad W \leq q \leq H / W,
$$

is bounded by $O(\log H / W H)$ as required.

\section{Proof of major arc estimate}

We now prove Proposition 2.4 in the major arc case $q \leq W$. We will discard the factor $d^{1 / 4}(\log H)^{1 / 4} \log \log H$ and prove the stronger bound

$$
\int_{\mathbb{R}}\left|\sum_{x / d \leq n \leq(x+H) / d} 1_{\mathcal{S}}(n) g(n) e(\alpha n)\right| d x \ll \frac{H X}{d W^{1 / 4}} .
$$

By hypothesis we have $\alpha=a / q+\theta$ with $q \leq W$ and $\theta=O(W /(H q))$. Integrating by parts we see that

$$
\begin{aligned}
& \left|\sum_{x / d \leq n \leq(x+H) / d} 1_{\mathcal{S}}(n) g(n) e(\alpha n)\right| \\
& \ll\left|\sum_{x / d \leq n \leq(x+H) / d} 1_{\mathcal{S}}(n) g(n) e(a n / q)\right| \\
& +\frac{W}{H q} \int_{0}^{H / d}\left|\sum_{x / d \leq n \leq x / d+H^{\prime}} 1_{\mathcal{S}}(n) g(n) e(a n / q)\right| d H^{\prime} .
\end{aligned}
$$

\footnotetext{
${ }^{4}$ For instance, from [Montgomery and Vaughan 2007, Theorem 3.13] one sees that any number $N=O(P)$ has $O\left((N / \phi(N))\left(P / \log ^{2} P\right)\right)$ representations as the sum of two primes; since $\sum_{N=O(P)} N^{2} / \phi(N)^{2}=O(P)$ (see, e.g., [Montgomery and Vaughan 2007, Exercise 2.1.14]), the claim then follows from the Cauchy-Schwarz inequality.
} 
Thus let us focus on bounding

$$
\int_{\mathbb{R}}\left|\sum_{x / d \leq n \leq x / d+H^{\prime}} 1_{\mathcal{S}}(n) g(n) e(a n / q)\right| d x
$$

with $0 \leq H^{\prime} \leq H / d$. Splitting into residues classes we see that (4-3) is

$$
\leq\left.\sum_{b(\bmod q)} \int_{\mathbb{R}}\right|_{\substack{x / d \leq n \leq x / d+H^{\prime} \\ n \equiv b(\bmod q)}} 1_{\mathcal{S}}(n) g(n) \mid d x
$$

For $n \equiv b(\bmod q)$ we have $d_{0}:=(b, q) \mid n$. Therefore let us write $b=d_{0} b_{0}, q=d_{0} q_{0}$ and $n=d_{0} m$, so that the condition $n \equiv b(\bmod q)$ simplifies to $m \equiv b_{0}\left(\bmod q_{0}\right)$. In addition, since $g$ is completely multiplicative and since $d_{0} \leq q \leq W \leq P_{1}$, we have

$$
1_{\mathcal{S}}(n) g(n)=g\left(d_{0}\right) \cdot 1_{\mathcal{S}_{P_{1}, Q_{1}, \sqrt{X}, X /\left(d d_{0}\right)}}(m) g(m) .
$$

Finally we express $m \equiv b_{0}\left(\bmod q_{0}\right)$ in terms of Dirichlet characters noting that

$$
1_{m \equiv b_{0}\left(\bmod q_{0}\right)}(m)=\frac{1}{\varphi\left(q_{0}\right)} \sum_{\chi\left(\bmod q_{0}\right)} \chi\left(b_{0}\right) \overline{\chi(m)} .
$$

Putting everything together we see that (4-3) is less than

$$
\left.\sum_{b(\bmod q)} \frac{1}{\varphi\left(q_{0}\right)} \sum_{\chi} \int_{\left(\bmod q_{0}\right)}\right|_{\mathbb{R}} \sum_{x /\left(d d_{0}\right) \leq m \leq x /\left(d d_{0}\right)+H^{\prime} / d_{0}} 1_{\mathcal{S}_{P_{1}, Q_{1}, \sqrt{X}, X /\left(d d_{0}\right)}} g(m) \overline{\chi(m)} \mid d x .
$$

In the integral we make the linear change of variable $y=x /\left(d d_{0}\right)$, so that the above expression becomes

$$
d \sum_{b(\bmod q)} \frac{d_{0}}{\varphi\left(q_{0}\right)} \sum_{\chi\left(\bmod q_{0}\right)} \int_{\mathbb{R}}\left|\sum_{y \leq m \leq y+H^{\prime} / d_{0}} 1_{\mathcal{S}_{P_{1}, Q_{1}, \sqrt{X}, X /\left(d d_{0}\right)}} g(m) \overline{\chi(m)}\right| d y .
$$

We bound the part of the integral with $y \leq X / W^{10}$ trivially. This produces in (4-3) an error which is

$$
\ll d q \cdot \frac{X}{W^{10}} \cdot H^{\prime} \leq \frac{H X}{W^{9}} \ll \frac{H X}{d W^{3}}
$$

since $q, d \leq W$ and $H^{\prime} \leq H / d$. We split the remaining range $X / W^{10} \leq y \leq 2 X /\left(d d_{0}\right)$ into dyadic blocks $X / W^{10} \leq X^{\prime} \leq X /\left(d d_{0}\right)$ with $X^{\prime}$ running through powers of two. Thus the previous expression is

$$
\ll d \sum_{X^{\prime}} \sum_{b(\bmod q)} \frac{d_{0}}{\varphi\left(q_{0}\right)} \sum_{\chi} \int_{\left(\bmod q_{0}\right)}^{2 X^{\prime}}\left|\sum_{y \leq m \leq y+H^{\prime} / d_{0}} 1_{\mathcal{S}_{P_{1}, Q_{1}, \sqrt{X}, X /\left(d d_{0}\right)}} g(m) \overline{\chi(m)}\right| d y+\frac{H X}{d W^{3}} .
$$


At this point we apply Theorem A.2 with $\eta=1 / 20$ (note that $\left.P_{1} \geq\left(\log Q_{1}\right)^{40 / \eta}\right)$ to conclude that

$$
\begin{aligned}
\int_{X^{\prime}}^{2 X^{\prime}} & \left.\sum_{y \leq m \leq y+H^{\prime} / d_{0}} 1_{\mathcal{S}_{P_{1}, Q_{1}, \sqrt{X}, X /\left(d d_{0}\right)}}(m) g(m) \bar{\chi}(m)\right|^{2} d y \\
& \ll\left(\exp \left(-M\left(g \bar{\chi} ; X^{\prime}\right)\right) M\left(g \bar{\chi} ; X^{\prime}\right)+\frac{\left(\log H^{\prime} / d_{0}\right)^{1 / 3}}{P_{1}^{1 / 6-1 / 20}}+\frac{1}{\left(\log X^{\prime}\right)^{1 / 50}}\right) \frac{H^{\prime 2}}{d_{0}^{2}} X^{\prime} .
\end{aligned}
$$

Since $P_{1}=W^{200}$ and $H^{\prime} / d_{0} \leq H$ and $W \geq \log ^{5} H$, we have

$$
\frac{\left(\log H^{\prime} / d_{0}\right)^{1 / 3}}{P_{1}^{1 / 6-1 / 20}} \leq \frac{(\log H)^{1 / 3}}{P_{1}^{1 / 6-1 / 20}} \ll \frac{1}{W^{5 / 2}}
$$

and certainly

$$
\frac{1}{\left(\log X^{\prime}\right)^{1 / 50}} \ll \frac{1}{(\log X)^{1 / 50}} \ll \frac{1}{W^{5 / 2}} .
$$

From Mertens' theorem and definition of $M(g, X, W)$,

$$
M\left(g \bar{\chi} ; X^{\prime}\right) \geq M(g \bar{\chi} ; X)-O(1) \geq M(g, X, W)-O(1)
$$

and thus, by (2-3),

$$
\exp \left(-M\left(g \bar{\chi} ; X^{\prime}\right)\right) M\left(g \bar{\chi} ; X^{\prime}\right) \ll \frac{1}{W^{5 / 2}} .
$$

Putting all this together, we obtain

$$
\int_{X^{\prime}}^{2 X^{\prime}}\left|\sum_{y \leq m \leq y+H^{\prime} / d_{0}, Q_{1}, \sqrt{X}, X /\left(d d_{0}\right)}(m) g(m) \bar{\chi}(m)\right|^{2} d y \ll \frac{1}{W^{5 / 2}} \frac{H^{\prime 2}}{d_{0}^{2}} X^{\prime} .
$$

It follows from Cauchy-Schwarz that

$$
\int_{X^{\prime}}^{2 X^{\prime}} \sum_{y \leq m \leq y+H^{\prime} / d_{0}} 1_{\mathcal{S}_{P_{1}, Q_{1}, \sqrt{X}, X /\left(d d_{0}\right)}}(m) g(m) \overline{\chi(m)} \mid d y \ll W^{-5 / 4} \cdot \frac{H^{\prime} X^{\prime}}{d_{0}} .
$$

Inserting this bound into (4-4) we see that (4-3) is bounded by

$$
\ll d q \cdot \frac{1}{W^{5 / 4}} \cdot \frac{H}{d} \cdot \frac{X}{d} \ll \frac{q H X}{d W^{5 / 4}} .
$$

Therefore using (4-2) and using $q \leq W$ we see that (4-1) is

$$
\ll \frac{q H X}{d W^{5 / 4}} \cdot\left(1+\frac{W}{H q} \cdot \frac{H}{d}\right) \ll \frac{H X}{d W^{1 / 4}} .
$$




\section{Elliott's conjecture on the average}

In this section we use Theorem 2.3 to prove Theorem 1.6 , which will be deduced from the following result (compare also with Theorem 2.3 and deduction of Theorem 1.7 from it). For brevity, we write $1_{\mathcal{S}} g$ for the function $n \mapsto 1_{\mathcal{S}}(n) g(n)$.

Proposition 5.1 (truncated Elliott on the average). Let $X, H, W, A \geq 10$ be such that

$$
\log ^{20} H \leq W \leq \min \left\{H^{1 / 500},(\log X)^{1 / 125}\right\} .
$$

Let $g_{1}, \ldots, g_{k}: \mathbb{N} \rightarrow \mathbb{C}$ be 1-bounded multiplicative functions, and let $a_{1}, \ldots, a_{k}$, $b_{1}, \ldots, b_{k}$ be natural numbers with $a_{j} \leq A$ and $b_{j} \leq 3 A X$ for $j=1, \ldots, k$. Let $1 \leq j_{0} \leq k$ be such that

$$
W \leq \exp \left(M\left(g_{j_{0}} ; 10 A X, Q\right) / 3\right) .
$$

Set

$$
\mathcal{S}=\mathcal{S}_{P_{1}, Q_{1}, \sqrt{10 A X}, 10 A X} \text { where } P_{1}:=W^{200}, Q_{1}:=H^{1 / 2} / W^{3} .
$$

Then

$$
\sum_{1 \leq h_{2}, \ldots, h_{k} \leq H}\left|\sum_{1 \leq n \leq X} 1_{\mathcal{S}} g_{1}\left(a_{1} n+b_{1}\right) \prod_{j=2}^{k} 1_{\mathcal{S}} g_{j}\left(a_{j} n+b_{j}+h_{j}\right)\right| \ll \frac{k A^{2}}{W^{1 / 20}} H^{k-1} X .
$$

Proof of Theorem 1.6 assuming Proposition 5.1. We may assume that $X, H$, and $M$ are larger than any specified absolute constant, as the claim is trivial otherwise. We first make some initial reductions. The first estimate (1-10) of Theorem 1.6 follows from the second (1-11) after shifting $b_{1}$ by $h_{1}$ in (1-11) and averaging, provided that we relax the hypotheses $b_{j} \leq A X$ slightly to $b_{j} \leq 2 A X$. Thus it suffices to prove (1-11) under the relaxed hypotheses $b_{j} \leq 2 A X$.

Let $H_{0}$ be such that

$\log H_{0}=\min \left\{\log ^{1 / 3000} X \log \log X, \exp \left(M\left(g_{j_{0}} ; 10 A X, Q\right) / 80\right) M\left(g_{j_{0}} ; 10 A X, Q\right)\right\}$.

If $H \leq H_{0}$ we take $W=\log ^{20} H$ and let $\mathcal{S}$ be as in Proposition 5.1. All the assumptions of Proposition 5.1 hold and thus

$$
\sum_{1 \leq h_{2}, \ldots, h_{k} \leq H}\left|\sum_{1 \leq n \leq X} 1_{\mathcal{S}} g_{1}\left(a_{1} n+b_{1}\right) \prod_{j=2}^{k} 1_{\mathcal{S}} g_{j}\left(a_{j} n+b_{j}+h_{j}\right)\right| \ll \frac{k A^{2}}{\log H} H^{k-1} X .
$$

Furthermore, from Lemma 2.2 we have

$$
\sum_{\substack{n \leq 10 A X \\ n \notin \mathcal{S}}} 1 \ll A X \frac{\log W}{\log H} .
$$


From this and the triangle inequality, we have

$$
\begin{aligned}
& \sum_{1 \leq n \leq X} g_{1}\left(a_{1} n+b_{1}\right) \prod_{j=2}^{k} g_{j}\left(a_{j} n+b_{j}+h_{j}\right) \\
& \quad=\sum_{1 \leq n \leq X} 1_{\mathcal{S}} g_{1}\left(a_{1} n+b_{1}\right) \prod_{j=2}^{k} 1_{\mathcal{S}} g_{j}\left(a_{j} n+b_{j}+h_{j}\right)+O\left(k A X \frac{\log W}{\log H}\right) .
\end{aligned}
$$

Hence the claim follows in the case when $H \leq H_{0}$.

If $H>H_{0}$, one can cover the summation over the $h_{j}$ indices by intervals of length $H_{0}$ and apply Theorem 1.6 to each subinterval (shifting the $b_{j}$ by at most $A X$ when doing so), and then sum, noting that the quantity

$$
\exp \left(-M\left(g_{j 0} ; 10 A X, Q\right) / 80\right)+\frac{\log \log H}{\log H}+\frac{1}{\log ^{1 / 3000} X}
$$

is essentially unchanged after replacing $H$ with $H_{0}$.

Remark 5.2. By using larger choices of $W$, one can obtain more refined information on the large values of the correlations $\sum_{1 \leq n \leq X} g_{1}\left(a_{1} n+b_{1}\right) \prod_{j=2}^{k} g_{j}\left(a_{j} n+b_{j}+h_{j}\right)$. For instance, if we take $W=H^{\delta}$ for some $H, \delta$ such that $10 \leq H \leq H_{0}$ and $20 \log \log H / \log H \leq \delta \leq 1 / 500$, we see from Proposition 5.1, (5-4), and Markov's inequality that

$$
\sum_{1 \leq n \leq X} g_{1}\left(a_{1} n+b_{1}\right) \prod_{j=2}^{k} g_{j}\left(a_{j} n+b_{j}+h_{j}\right) \ll k A^{2} \delta X
$$

for all but at most $O\left(H^{k-1} / \delta H^{\delta / 20}\right)$ tuples $\left(h_{1}, \ldots, h_{k-1}\right)$ with $1 \leq h_{j} \leq H$ for $j=2, \ldots, k$. Thus we can obtain a power saving in the number of exceptional tuples, at the cost of only obtaining a weak bound on the individual correlations $\sum_{1 \leq n \leq X} g_{1}\left(a_{1} n+b_{1}\right) \prod_{j=2}^{k} g_{j}\left(a_{j} n+b_{j}+h_{j}\right)$.

It remains to prove Proposition 5.1. We start by proving the following simpler case to which the general case will be reduced.

Proposition 5.3. Let $X, H, W \geq 10$ be such that

$$
\log ^{20} H \leq W \leq \min \left\{H^{1 / 250},(\log X)^{1 / 125}\right\} .
$$

Let $g: \mathbb{N} \rightarrow \mathbb{C}$ be a 1-bounded multiplicative function such that

$$
W \leq \exp (M(g ; X, W) / 3) .
$$

Set

$$
\mathcal{S}=\mathcal{S}_{P_{1}, Q_{1}, \sqrt{X}, X} \text { where } P_{1}:=W^{200}, Q_{1}:=H / W^{3} \text {. }
$$


Then

$$
\sum_{1 \leq h \leq H}\left|\sum_{1 \leq n \leq X} 1_{\mathcal{S}} g(n) 1_{\mathcal{S}} \bar{g}(n+h)\right|^{2} \ll \frac{H X^{2}}{W^{1 / 5}} .
$$

To deduce Theorem 1.2 we let $\mathcal{S}$ be as in this proposition with $W:=H^{\delta / 900}$. The argument of Lemma 2.2 actually gives $\#\{1 \leq n \leq X: n \notin \mathcal{S}\} \leq 2 X \log P_{1} / \log Q_{1}$ in this case, and thus the numbers $n$ with $n \notin \mathcal{S}$ or $n+h \notin \mathcal{S}$ contribute to the left-hand side of (1-4) at most $9 \delta / 10$. Hence, recalling (1-12), the claim follows from the previous proposition and Markov's inequality.

Proof of Proposition 5.3. The claim follows once we have shown

$$
\sum_{|h| \leq 2 H}(2 H-|h|)^{2} \cdot\left|\sum_{n} 1_{\mathcal{S}} g(n) 1_{\mathcal{S}} \bar{g}(n+h)\right|^{2} \ll \frac{1}{W^{1 / 5}} H^{3} X^{2} .
$$

Applying Lemma 1.4, it will suffice to show that

$$
\int_{\mathbb{T}}\left(\left.\left.\int_{\mathbb{R}}\right|_{x \leq n \leq x+2 H} 1_{\mathcal{S}} g(n) e(\alpha n)\right|^{2} d x\right)^{2} d \alpha \ll \frac{1}{W^{1 / 5}} H^{3} X^{2} .
$$

From the Parseval identity we have

$$
\int_{\mathbb{T}} \int_{\mathbb{R}}\left|\sum_{x \leq n \leq x+2 H} 1_{\mathcal{S}} g(n) e(\alpha n)\right|^{2} d x d \alpha=\int_{\mathbb{R}} \sum_{x \leq n \leq x+2 H}\left|1_{\mathcal{S}} g(n)\right|^{2} d x \ll H X
$$

so it suffices to show that

$$
\sup _{\alpha} \int_{\mathbb{R}}\left|\sum_{x \leq n \leq x+2 H} 1_{\mathcal{S}} g(n) e(\alpha n)\right|^{2} d x \ll \frac{1}{W^{1 / 5}} H^{2} X .
$$

Using the trivial bound

$$
\left|\sum_{x \leq n \leq x+2 H} 1_{\mathcal{S}} g(n) e(\alpha n)\right| \ll H
$$

we thus reduce to showing

$$
\sup _{\alpha} \int_{\mathbb{R}}\left|\sum_{x \leq n \leq x+2 H} 1_{\mathcal{S}} g(n) e(\alpha n)\right| d x \ll \frac{H X}{W^{1 / 5}} .
$$

This follows from Theorem 2.3 (using the lower bound $W \geq \log ^{20} H$ in the hypotheses of Proposition 5.3 to absorb the $\log ^{1 / 4} H \log \log H$ factors in Theorem 2.3).

Proof of Proposition 5.1. We first remove the special treatment afforded to the $g_{1}$ factor in (5-1). Note that we may assume

$$
W^{1 / 20} \geq k A^{2}
$$


and thus

$$
H \geq W^{500} \geq\left(k A^{2}\right)^{10000}
$$

since the claim is trivial otherwise.

Set $H^{\prime}:=\sqrt{H}$. For any $1 \leq h_{1} \leq H^{\prime} / A$, we may shift $n$ by $h_{1}$ and conclude that

$$
\begin{aligned}
& \sum_{1 \leq n \leq X} 1_{\mathcal{S}} g_{1}\left(a_{1} n+b_{1}\right) \prod_{j=2}^{k} 1_{\mathcal{S}} g_{j}\left(a_{j} n+b_{j}+h_{j}\right) \\
&=\sum_{1 \leq n \leq X} 1_{\mathcal{S}} g_{1}\left(a_{1} n+b_{1}+a_{1} h_{1}\right) \prod_{j=2}^{k} 1_{\mathcal{S}} g_{j}\left(a_{j} n+b_{j}+h_{j}+a_{j} h_{1}\right)+O\left(H^{\prime}\right)
\end{aligned}
$$

and thus we may write the left-hand side of (5-1) as

$$
\begin{array}{r}
\sum_{1 \leq h_{2}, \ldots, h_{k} \leq H}\left|\sum_{1 \leq n \leq X} 1_{\mathcal{S}} g_{1}\left(a_{1} n+b_{1}+a_{1} h_{1}\right) \prod_{j=2}^{k} 1_{\mathcal{S}} g_{j}\left(a_{j} n+b_{j}+h_{j}+a_{j} h_{1}\right)\right| \\
+O\left(H^{k-1} H^{\prime}\right) .
\end{array}
$$

If one shifts each of the $h_{j}$ for $j=2, \ldots, k$ in turn by $a_{j} h_{1}=O\left(H^{\prime}\right)$, we may rewrite this as

$$
\begin{array}{r}
\sum_{1 \leq h_{2}, \ldots, h_{k} \leq H}\left|\sum_{1 \leq n \leq X} 1_{\mathcal{S}} g_{1}\left(a_{1} n+b_{1}+a_{1} h_{1}\right) \prod_{j=2}^{k} 1_{\mathcal{S}} g_{j}\left(a_{j} n+b_{j}+h_{j}\right)\right| \\
+O\left(H^{k-1} H^{\prime}\right)+O\left(k H^{k-2} H^{\prime} X\right) .
\end{array}
$$

Averaging in $h_{1}$, and replacing $h_{1}$ by $a_{1} h_{1}$ (crudely dropping the constraint that $a_{1} h_{1}$ is divisible by $a_{1}$ ), we may thus bound the left-hand side of (5-1) by

$$
\begin{array}{r}
\ll \frac{A}{H^{\prime}} \sum_{1 \leq h_{1} \leq H^{\prime}} \sum_{1 \leq h_{2}, \ldots, h_{k} \leq H}\left|\sum_{1 \leq n \leq X} 1_{\mathcal{S}} g_{1}\left(a_{1} n+b_{1}+h_{1}\right) \prod_{j=2}^{k} 1_{\mathcal{S}} g_{j}\left(a_{j} n+b_{j}+h_{j}\right)\right| \\
+H^{k-1} H^{\prime}+k H^{k-2} H^{\prime} X .
\end{array}
$$

The $g_{1}$ term may now be combined with the product over the remaining $g_{j}$ terms to form $\prod_{j=1}^{k} 1_{\mathcal{S}} g_{j}\left(a_{j} n+b_{j}+h_{j}\right)$. The error term $H^{k-1} H^{\prime}+k H^{k-2} H^{\prime} X$ is certainly of size $O\left(\left(k A^{2} / W^{1 / 20}\right) H^{k-1} X\right)$, so it suffices to show that

$$
\sum_{1 \leq h_{1} \leq H^{\prime}} \sum_{1 \leq h_{2}, \ldots, h_{k} \leq H}\left|\sum_{1 \leq n \leq X} \prod_{j=1}^{k} 1_{\mathcal{S}} g_{j}\left(a_{j} n+b_{j}+h_{j}\right)\right| \ll \frac{A}{W^{1 / 20}} H^{k-1} H^{\prime} X .
$$


By covering the ranges $1 \leq h_{j} \leq H$ by intervals of length $H^{\prime}$ and averaging, it suffices (after relaxing the conditions $b_{j} \leq 3 A X$ to $b_{j} \leq 4 A X$ ) to prove that

$$
\sum_{1 \leq h_{1}, h_{2}, \ldots, h_{k} \leq H^{\prime}}\left|\sum_{1 \leq n \leq X} \prod_{j=1}^{k} 1_{\mathcal{S}} g_{j}\left(a_{j} n+b_{j}+h_{j}\right)\right| \ll \frac{A}{W^{1 / 20}}\left(H^{\prime}\right)^{k} X .
$$

The situation is now symmetric with respect to permuting the indices $1, \ldots, k$, so we may assume that the index $j_{0}$ in Proposition 5.1 is equal to 1 . By the triangle inequality in $h_{2}, \ldots, h_{k}$, it suffices to show that

$$
\sum_{1 \leq h_{1} \leq H^{\prime}}\left|\sum_{1 \leq n \leq X} \prod_{j=1}^{k} 1_{\mathcal{S}} g_{j}\left(a_{j} n+b_{j}+h_{j}\right)\right| \ll \frac{A}{W^{1 / 20}} H^{\prime} X
$$

for all $h_{2}, \ldots, h_{k}$. Writing $G(n):=\prod_{j=2}^{k} 1_{\mathcal{S}} g_{j}\left(a_{j} n+b_{j}+h_{j}\right)$, it thus suffices to show that

$$
\sum_{1 \leq h_{1} \leq H^{\prime}}\left|\sum_{1 \leq n \leq X} 1_{\mathcal{S}} g_{1}\left(a_{1} n+b_{1}+h_{1}\right) G(n)\right| \ll \frac{A}{W^{1 / 20}} H^{\prime} X
$$

for any 1-bounded function $G: \mathbb{Z} \rightarrow \mathbb{C}$.

We use a standard van der Corput argument. By the Cauchy-Schwarz inequality, it suffices to show that

$$
\sum_{1 \leq h_{1} \leq H^{\prime}}\left|\sum_{1 \leq n \leq X} 1_{\mathcal{S}} g_{1}\left(a_{1} n+b_{1}+h_{1}\right) G(n)\right|^{2} \ll \frac{A^{2}}{W^{1 / 10}}\left(H^{\prime}\right)^{2} X^{2} .
$$

The left-hand side may be rewritten as

$$
\sum_{n, n^{\prime} \leq X} G(n) \overline{G\left(n^{\prime}\right)} \sum_{1 \leq h_{1} \leq H^{\prime}} 1_{\mathcal{S}} g_{1}\left(a_{1} n+b_{1}+h_{1}\right) 1_{\mathcal{S}} \overline{g_{j}}\left(a_{1} n^{\prime}+b_{1}+h_{1}\right) .
$$

By the triangle inequality, it thus suffices to show that

$$
\sum_{n, n^{\prime} \leq X}\left|\sum_{1 \leq h_{1} \leq H^{\prime}} 1_{\mathcal{S}} g_{1}\left(a_{1} n+b_{1}+h_{1}\right) 1_{\mathcal{S}} \overline{g_{1}}\left(a_{1} n^{\prime}+b_{1}+h_{1}\right)\right| \ll \frac{A^{2}}{W^{1 / 10}} H^{\prime} X^{2} .
$$

To abbreviate notation we now write $h=h_{1}, g=g_{1}, a=a_{1}, b=b_{1}$. By the Cauchy-Schwarz inequality, it suffices to show that

$$
\sum_{n, n^{\prime} \leq X}\left|\sum_{1 \leq h \leq H^{\prime}} 1_{\mathcal{S}} g(a n+b+h) 1_{\mathcal{S}} \bar{g}\left(a n^{\prime}+b+h\right)\right|^{2} \ll \frac{A^{4}}{W^{1 / 5}}\left(H^{\prime}\right)^{2} X^{2} .
$$


Replacing $n, n^{\prime}$ by $a n+b, a n^{\prime}+b$ respectively, it suffices to show that

$$
\sum_{n, n^{\prime}}\left|\sum_{1 \leq h \leq H^{\prime}} 1_{\mathcal{S}} g(n+h) 1_{\mathcal{S}} \bar{g}\left(n^{\prime}+h\right)\right|^{2} \ll \frac{A^{4}}{W^{1 / 5}}\left(H^{\prime}\right)^{2} X^{2}
$$

where we have extended $1_{\mathcal{S}} g$ by zero to the negative integers. The left-hand side can be rewritten as

$$
\sum_{|h|<H^{\prime}}\left(\left\lfloor H^{\prime}\right\rfloor-|h|\right)\left|\sum_{n} 1_{\mathcal{S}} g(n) 1_{\mathcal{S}} \bar{g}(n+h)\right|^{2},
$$

and the claim follows from Proposition 5.3.

\section{Appendix A: Mean values of complex multiplicative functions in short intervals}

In this section we prove a complex variant of results in [Matomäki and Radziwiłł 2015 ] in the case that $f$ is not $p^{i t}$ pretentious. In particular, we show that the mean value of a 1-bounded nonpretentious multiplicative function is small for most short intervals:

Theorem A.1. Let $f$ be a 1-bounded multiplicative function and let $M(f ; X)$ be as in (1-6). Then, for $X \geq h \geq 10$,

$$
\frac{1}{X} \int_{X}^{2 X}\left|\frac{1}{h} \sum_{x \leq n \leq x+h} f(n)\right|^{2} d x \ll \exp (-M(f ; X)) M(f ; X)+\frac{(\log \log h)^{2}}{(\log h)^{2}}+\frac{1}{(\log X)^{1 / 50}} .
$$

Actually, as in [Matomäki and Radziwiłł 2015] and earlier in this paper, one gets better quantitative results if one first restricts to a subset of $n$ with a typical factorization. Let us first define such a subset $\mathcal{S}$ in this setting.

Let $\eta \in(0,1 / 6)$, and let $X_{0}$ be a quantity with $\sqrt{X} \leq X_{0} \leq X$. (The results in [Matomäki and Radziwiłł 2015] used the choice $X_{0}=X$, but for technical reasons we will need a more flexible choice of this parameter.) Consider a sequence of increasing intervals $\left[P_{j}, Q_{j}\right], j \geq 1$ such that:

- $Q_{1} \leq \exp \left(\sqrt{\log X_{0}}\right)$.

- The intervals are not too far from each other; precisely, for all $j \geq 2$,

$$
\frac{\log \log Q_{j}}{\log P_{j-1}-1} \leq \frac{\eta}{4 j^{2}}
$$

- The intervals are not too close to each other; precisely, for all $j \geq 2$,

$$
\frac{\eta}{j^{2}} \log P_{j} \geq 8 \log Q_{j-1}+16 \log j
$$


For example, given $0<\eta<1 / 6$, the sequence of intervals $\left[P_{j}, Q_{j}\right]$ defined in Definition 2.1 can be verified to obey the above estimates if

$$
\exp \left(\sqrt{\log X_{0}}\right) \geq Q_{1} \geq P_{1} \geq\left(\log Q_{1}\right)^{40 / \eta}
$$

and if $P_{1}$ is sufficiently large.

Let $\mathcal{S}$ be the set of integers $X \leq n \leq 2 X$ having at least one prime factor in each of the intervals $\left[P_{j}, Q_{j}\right]$ for $j \leq J$, where $J$ is chosen to be the largest index $j$ such that $Q_{j} \leq \exp \left(\left(\log X_{0}\right)^{1 / 2}\right)$. We will establish the following variant of [Matomäki and Radziwiłł 2015, Theorem 3].

Theorem A.2. Let $f$ be a 1-bounded multiplicative function. Let $\mathcal{S}$ be as above with $\eta \in(0,1 / 6)$. If $\left[P_{1}, Q_{1}\right] \subset[1, h]$, then for all $X>X(\eta)$ large enough and all $h \geq 3$,

$$
\frac{1}{X} \int_{X}^{2 X}\left|\frac{1}{h} \sum_{\substack{x \leq n \leq x+h \\ n \in \mathcal{S}}} f(n)\right|^{2} d x \ll \exp (-M(f ; X)) M(f ; X)+\frac{(\log h)^{1 / 3}}{P_{1}^{1 / 6-\eta}}+\frac{1}{(\log X)^{1 / 50}} .
$$

The proof of Theorem A.2 proceeds as the proof of [Matomäki and Radziwiłł 2015, Theorem 3]. The first step is a Parseval bound:

$$
\frac{1}{X} \int_{X}^{2 X}\left|\frac{1}{h} \sum_{\substack{x \leq n \leq x+h \\ n \in \mathcal{S}}} f(n)\right|^{2} d x \ll \int_{1}^{1+i X / h_{1}}|F(s)|^{2}|d s|+\max _{T \geq X / h_{1}} \frac{X / h_{1}}{T} \int_{1+i T}^{1+i 2 T}|F(s)|^{2}|d s| .
$$

This follows exactly in the same way as [Matomäki and Radziwiłł 2015, Lemma 14] but there is no need to split the integral into two parts, and one can just work as for $V(x)$ there. Theorem A.2 now follows immediately from the following variant of [Matomäki and Radziwiłł 2015, Proposition 1].

Proposition A.3. Let $f$ be a 1-bounded multiplicative function. Let $\mathcal{S}$ be as above, and let

$$
F(s)=\sum_{\substack{X \leq n \leq 2 X \\ n \in \mathcal{S}}} \frac{f(n)}{n^{s}}
$$

Then, for any $T$,

$$
\begin{aligned}
& \int_{0}^{T}|F(1+i t)|^{2} d t \\
& \quad \ll\left(\frac{T}{X / Q_{1}}+1\right)\left(\frac{\left(\log Q_{1}\right)^{1 / 3}}{P_{1}^{1 / 6-\eta}}+\exp (-M(f ; X)) M(f ; X)+\frac{1}{(\log X)^{1 / 50}}\right) .
\end{aligned}
$$

Proof. Since the mean value theorem gives the bound $O(T / X+1)$, we can assume $T \leq X / 2$ and $M(f ; X) \geq 1$. 
Now let $t_{1}$ be the value of $t$ which attains the minimum in

$$
M(f ; X)=\inf _{|t| \leq X} \mathbb{D}\left(g, n \mapsto n^{i t} ; X\right)^{2} .
$$

We split the integration into three ranges:

$$
\begin{aligned}
& \mathcal{T}_{0}=\left\{0 \leq t \leq T:\left|t-t_{1}\right| \leq \exp (M(f ; X)) / M(f ; X)\right\}, \\
& \mathcal{T}_{1}=\left\{0 \leq t \leq T: \exp (M(f ; X)) / M(f ; X) \leq\left|t-t_{1}\right| \leq(\log X)^{1 / 16}\right\}, \\
& \mathcal{T}_{2}=\left\{0 \leq t \leq T:\left|t-t_{1}\right| \geq(\log X)^{1 / 16}\right\} .
\end{aligned}
$$

Notice that by the definition of $t_{1}$, the triangle inequality and arguing as in (1-12), for any $|t| \leq X$ with $\left|t-t_{1}\right| \geq 1$, and any $\varepsilon>0$,

$$
\begin{aligned}
2 \mathbb{D}\left(f, p^{i t} ; X\right) & \geq \mathbb{D}\left(f, p^{i t} ; X\right)+\mathbb{D}\left(f, p^{i t_{1}} ; X\right) \geq \mathbb{D}\left(1, p^{i\left(t-t_{1}\right)}\right) \\
& \geq\left(\frac{1}{\sqrt{3}}-\varepsilon\right) \sqrt{\log \log X}+O(1),
\end{aligned}
$$

so that by Halasz's theorem, for every $|t| \leq T$,

$$
F(1+i t) \ll(\log X)^{-1 / 16}+\frac{1}{1+\left|t-t_{1}\right|} .
$$

In the region $\left|t-t_{1}\right| \geq(\log X)^{1 / 16}$, the above implies the following in exactly the same way as [Matomäki and Radziwiłł 2015, Lemma 3].

Lemma A.4. Let $X \geq Q \geq P \geq 2$. Let $t_{1}$ be as above and let

$$
G(s)=\sum_{X \leq n \leq 2 X} \frac{f(n)}{n^{s}} \cdot \frac{1}{\#\{p \in[P, Q]: p \mid n\}+1} .
$$

Then, for any $t \in \mathcal{T}_{2}$,

$$
|G(1+i t)| \ll \frac{\log Q}{(\log X)^{1 / 16} \log P}+\log X \cdot \exp \left(-\frac{\log X}{3 \log Q} \log \frac{\log X}{\log Q}\right) .
$$

This was the only part in the proof [Matomäki and Radziwiłł 2015, Proposition 1] that needed $f$ to be real-valued, and thus we get

$$
\int_{\mathcal{T}_{2}}|F(1+i t)|^{2} d t \ll\left(\frac{T}{X / Q_{1}}+1\right)\left(\frac{\left(\log Q_{1}\right)^{1 / 3}}{P_{1}^{1 / 6-\eta}}+\frac{1}{(\log X)^{1 / 50}}\right) .
$$

Using the estimate $F(1+i t) \ll 1 /\left|t-t_{1}\right|$ for $t \in \mathcal{T}_{1}$ and, from Halasz's theorem, the estimate $F(1+i t) \ll \exp (-M(f ; X)) M(f ; X)$ for $t \in \mathcal{T}_{0}$, we obtain

$$
\int_{\mathcal{T}_{0} \cup \mathcal{T}_{1}}|F(1+i t)|^{2} d t \ll \exp (-M(f ; X)) M(f ; X),
$$

and the claim follows. 
Proof of Theorem A.1. Let $\eta=1 / 12, P_{1}=(\log h)^{480}, Q_{1}=h$, let $P_{j}$ and $Q_{j}$ for $j \geq 2$ be as in Definition 2.1, and let $\mathcal{S}$ be as above. Then

$$
\frac{1}{X} \int_{X}^{2 X}\left|\frac{1}{h} \sum_{x \leq n \leq x+h} f(n)\right|^{2} d x \leq \frac{1}{X} \int_{X}^{2 X}\left|\frac{1}{h} \sum_{\substack{x \leq n \leq x+h \\ n \in \mathcal{S}}} f(n)\right|^{2} d x+\frac{1}{X} \int_{X}^{2 X}\left|\frac{1}{h} \sum_{\substack{x \leq n \leq x+h \\ n \notin \mathcal{S}}} 1\right|^{2} d x .
$$

The contribution from the first integral is acceptable by Theorem A.2. We rewrite the second integrand as

$$
\begin{aligned}
\left|\frac{1}{h} \sum_{\substack{x \leq n \leq x+h \\
n \notin \mathcal{S}}} 1\right| & =\left|1+O(1 / h)-\frac{1}{h} \sum_{\substack{x \leq n \leq x+h \\
n \in \mathcal{S}}} 1\right| \\
& \leq\left|\frac{1}{X} \sum_{\substack{X \leq n \leq 2 X \\
n \in \mathcal{S}}} 1-\frac{1}{h} \sum_{\substack{x \leq n \leq x+h \\
n \in \mathcal{S}}} 1\right|+\left|\frac{1}{X} \sum_{\substack{X \leq n \leq 2 X \\
n \notin \mathcal{S}}} 1\right|+O(1 / h),
\end{aligned}
$$

and the claim follows from [Matomäki and Radziwiłł 2015, Theorem 3 with $f=1$ ] and Lemma 2.2.

\section{Appendix B: Counterexample to the uncorrected Elliott conjecture}

In this appendix we present a counterexample to Conjecture 1.5. More precisely:

Theorem B.1 (counterexample). There exists a 1-bounded multiplicative function $g: \mathbb{N} \rightarrow \mathbb{C}$ such that

$$
\sum_{p} \frac{1-\operatorname{Re}\left(g(p) \overline{\chi(p)} p^{-i t}\right)}{p}=\infty
$$

for all Dirichlet characters $\chi$ and $t \in \mathbb{R}$ (i.e., one has $M(g ; \infty, \infty)=\infty)$, but such that

$$
\left|\sum_{n \leq t_{m}} g(n) \overline{g(n+1)}\right| \gg t_{m}
$$

for all sufficiently large $m$ and some sequence $t_{m}$ going to infinity.

Proof. For each prime $p$, we choose $g(p)$ from the unit circle $S^{1}:=\{z:|z|=1\}$ by the following iterative procedure involving a sequence $t_{1}<t_{2}<t_{3}<\cdots$ :

(1) Initialize $t_{1}:=100$ and $m:=1$, and set $g(p):=1$ for all $p \leq t_{1}$.

(2) Now suppose recursively that $g(p)$ has been chosen for all $p \leq t_{m}$. As the quantities $\log p$ are linearly independent over the integers, the (continuous) sequence $t \mapsto(t \log p \bmod 1)_{p \leq t_{m}}$ is equidistributed in the torus $\prod_{p \leq t_{m}} \mathbb{T}$ and, equivalently, the sequence $t \mapsto\left(p^{i t}\right)_{p \leq t_{m}}$ is equidistributed in the torus $\prod_{p \leq t_{m}} S^{1}$. 
Thus one can find a quantity $s_{m+1}>\exp \left(t_{m}\right)$ such that, for all $p \leq t_{m}$,

$$
p^{i s_{m+1}}=g(p)\left(1+O\left(\frac{1}{t_{m}^{2}}\right)\right) .
$$

(3) Set $t_{m+1}:=s_{m+1}^{2}$, and then set

$$
g(p):=p^{i s_{m+1}}
$$

for all $t_{m}<p \leq t_{m+1}$. Now increment $m$ to $m+1$ and return to step (2).

Clearly the $t_{m}$ go to infinity, so $g(p)$ is defined for all primes $p$. We then define

$$
g(n):=\mu(n)^{2} \prod_{p \mid n} g(p),
$$

which is clearly a 1-bounded multiplicative function.

Suppose that $n \leq t_{m+1}$ is squarefree. Then $n$ is the product of distinct primes less than or equal to $t_{m+1}$, including at most $t_{m}$ primes less than or equal to $t_{m}$. From (B-5) we then have

$$
g(n)=n^{i s_{m+1}}\left(1+O\left(\frac{1}{t_{m}^{2}}\right)\right)^{O\left(t_{m}\right)}=n^{i s_{m+1}}+O\left(\frac{1}{t_{m}}\right) .
$$

If $n$ is not squarefree, then $g(n)$ of course vanishes. Thus, for $t_{m+1}^{3 / 4} \leq n \leq t_{m+1}-1$, we have

$$
\begin{aligned}
g(n) \overline{g(n+1)} & =\mu^{2}(n) \mu^{2}(n+1)\left(\frac{n+1}{n}\right)^{i s_{m+1}}+O\left(\frac{1}{t_{m}}\right) \\
& =\mu^{2}(n) \mu^{2}(n+1)+O\left(\frac{s_{m+1}}{t_{m+1}^{3 / 4}}\right)+O\left(\frac{1}{t_{m}}\right) \\
& =\mu^{2}(n) \mu^{2}(n+1)+O\left(\frac{1}{t_{m}}\right),
\end{aligned}
$$

and the claim (B-2) then easily follows since the sequence $\mu^{2}(n) \mu^{2}(n+1)$ has positive mean value.

Now we prove (B-1). From (B-4), we have

$$
\begin{aligned}
\sum_{p} \frac{1-\operatorname{Re}\left(g(p) \overline{\chi(p)} p^{-i t}\right)}{p} & \geq \sum_{t_{m}<p \leq t_{m+1}} \frac{1-\operatorname{Re}\left(\overline{\chi(p)} p^{i\left(s_{m+1}-t\right)}\right)}{p} \\
& \geq \sum_{\exp \left(\left(\log t_{m+1}\right)^{5 / 6}\right)<p \leq t_{m+1}} \frac{1-\operatorname{Re}\left(\overline{\chi(p)} p^{i\left(s_{m+1}-t\right)}\right)}{p}
\end{aligned}
$$

since $\exp \left(\left(\log t_{m+1}\right)^{5 / 6}\right) \geq \exp \left(\left(2 t_{m}\right)^{5 / 6}\right) \geq t_{m}$. We see as in (1-12) that the righthand side goes to infinity as $m \rightarrow \infty$ for any fixed $\chi, t$, and the claim follows. 
It is easy to see that the function $g$ constructed in the above counterexample violates (1-9), and so is not a counterexample to the corrected form of Conjecture 1.5. It is also not difficult to modify the above counterexample so that the function $g$ is completely multiplicative instead of multiplicative, using the fact that most numbers up to $t_{m+1}$ have fewer than $t_{m}$ prime factors less than $t_{m}$ (counting multiplicity); we leave the details to the interested reader.

\section{Appendix C: An argument of Granville and Soundararajan}

In this appendix we show the equivalence of the hypotheses (1-7) and (1-9) for Elliott's conjecture in the case that the multiplicative function $g_{j_{0}}$ is real. The key lemma is the following estimate, essentially due to Granville and Soundararajan.

Lemma C.1. Let $f: \mathbb{N} \rightarrow[-1,1]$ be a multiplicative function, let $x \geq 100$, and let $\chi$ be a fixed Dirichlet character. For $1 \leq|\alpha| \leq x$, one has

$$
\mathbb{D}\left(f, n \mapsto \chi(n) n^{i \alpha} ; x\right) \geq \frac{1}{4} \sqrt{\log \log x}+O_{\chi}(1) .
$$

When $\chi^{2}$ is nonprincipal, this holds for all $|\alpha| \leq x$.

If $\chi^{2}$ is principal (i.e., $\chi$ is a quadratic character), then, for $|\alpha| \leq 1$, one has

$$
\mathbb{D}\left(f, n \mapsto \chi(n) n^{i \alpha} ; x\right) \geq \frac{1}{3} \mathbb{D}(f, \chi ; x)+O(1) .
$$

Proof. To establish (C-1), we notice that, by conjugation symmetry and the triangle inequality,

$$
\begin{aligned}
\mathbb{D}\left(f, n \mapsto \chi(n) n^{i \alpha} ; x\right) & =\frac{1}{2}\left(\mathbb{D}\left(f, n \mapsto \chi(n) n^{i \alpha} ; x\right)+\mathbb{D}\left(f, n \mapsto \overline{\chi(n)} n^{-i \alpha} ; x\right)\right) \\
& \geq \frac{1}{2} \mathbb{D}\left(n \mapsto \overline{\chi(n)} n^{-i \alpha}, n \mapsto \chi(n) n^{i \alpha} ; x\right) \\
& =\frac{1}{2}\left(\sum_{p \leq x} \frac{1-\operatorname{Re} \chi^{2}(p) p^{2 i \alpha}}{p}\right)^{1 / 2}
\end{aligned}
$$

which implies the claim for $|\alpha| \geq 1$ or for nonprincipal $\chi^{2}$ by the zero-free (and pole-free) region for Dirichlet $L$-functions (see (1-12) for a related argument).

To establish (C-2), notice first that since $\chi^{2}$ is principal, $\chi$ is real-valued which together with the triangle inequality implies

$\mathbb{D}\left(f, n \mapsto \chi(n) n^{i \alpha} ; x\right)=\mathbb{D}\left(f \chi, n \mapsto n^{i \alpha} ; x\right) \geq \mathbb{D}(1, f \chi ; x)-\mathbb{D}\left(1, n \mapsto n^{i \alpha} ; x\right)$.

Now $\mathbb{D}\left(1, n \mapsto n^{i \alpha} ; x\right)=\mathbb{D}\left(1, n \mapsto n^{2 i \alpha} ; x\right)+O(1)$ for $|\alpha| \leq 1$ since, from the prime number theorem, $\mathbb{D}\left(1, n \mapsto n^{i \alpha} ; x\right)^{2}=\log (1+|\alpha| \log x)+O(1)$, so that the 
claim follows unless $\mathbb{D}\left(1, n \mapsto n^{2 i \alpha} ; x\right) \geq(2 / 3) \mathbb{D}(1, f \chi ; x)$. But in the latter case, the triangle inequality gives

$$
\begin{aligned}
\frac{2}{3} \mathbb{D}(f, \chi ; x) & =\frac{2}{3} \mathbb{D}(1, f \chi ; x) \\
& \leq \mathbb{D}\left(1, n \mapsto n^{2 i \alpha} ; x\right) \\
& =\mathbb{D}\left(n \mapsto n^{-i \alpha}, n \mapsto n^{i \alpha} ; x\right) \\
& \leq \mathbb{D}\left(f \chi, n \mapsto n^{-i \alpha} ; x\right)+\mathbb{D}\left(f \chi ; n \mapsto n^{i \alpha} ; x\right) \\
& =2 \mathbb{D}\left(f, n \mapsto \chi(n) n^{i \alpha} ; x\right),
\end{aligned}
$$

and the claim (C-2) follows.

From this lemma, we see that if $g_{j_{0}}$ is a real 1-bounded multiplicative function, then, for given $Q$, the condition (1-9) is equivalent to

$$
\mathbb{D}\left(g_{j_{0}}, \chi ; X\right) \rightarrow \infty
$$

when $X \rightarrow \infty$ for all quadratic characters $\chi$ of modulus at most $Q$. But this follows from (1-7). The converse implication is trivial.

\section{Acknowledgments}

Tao was supported by a Simons Investigator grant, the James and Carol Collins Chair, the Mathematical Analysis \& Application Research Fund Endowment, and NSF grant DMS-1266164. The authors thank Andrew Granville and the anonymous referee for useful comments and corrections.

\section{References}

[Balog 1990] A. Balog, "The prime $k$-tuplets conjecture on average", pp. 47-75 in Analytic number theory (Allerton Park, IL, 1989), edited by B. C. Berndt et al., Progr. Math. 85, Birkhäuser, Boston, 1990. MR 92e:11105 Zbl 0719.11066

[Bourgain et al. 2013] J. Bourgain, P. Sarnak, and T. Ziegler, "Disjointness of Moebius from horocycle flows", pp. 67-83 in From Fourier analysis and number theory to radon transforms and geometry, edited by H. M. Farkas et al., Dev. Math. 28, Springer, New York, 2013. MR 2986954 Zbl 06150358

[Chowla 1965] S. Chowla, The Riemann hypothesis and Hilbert's tenth problem, Mathematics and Its Applications 4, Gordon and Breach Science Publishers, New York-London-Paris, 1965. MR 31 \#2201 Zbl 0133.30003

[Daboussi and Delange 1982] H. Daboussi and H. Delange, "On multiplicative arithmetical functions whose modulus does not exceed one”, J. London Math. Soc. (2) 26:2 (1982), 245-264. MR 83k:10082 Zbl 0499.10052

[Davenport 1937] H. Davenport, "On some infinite series involving arithmetical functions, II", Quart. J. Math. Oxf. 8 (1937), 313-320. Zbl 0017.39101

[Elliott 1992] P. D. T. A. Elliott, "On the correlation of multiplicative functions", Notas Soc. Mat. Chile 11:1 (1992), 1-11. MR 95j:11076 Zbl 0810.11055 
[Frantzikinakis and Host 2015] N. Frantzikinakis and B. Host, "Asymptotics for multilinear averages of multiplicative functions", preprint, 2015. arXiv 1502.02646

[Friedlander and Iwaniec 2010] J. Friedlander and H. Iwaniec, Opera de cribro, American Mathematical Society Colloquium Publications 57, American Mathematical Society, Providence, RI, 2010. MR 2011d:11227 Zbl 1226.11099

[Granville and Soundararajan 2007] A. Granville and K. Soundararajan, "Large character sums: pretentious characters and the Pólya-Vinogradov theorem", J. Amer. Math. Soc. 20:2 (2007), 357384. MR 2007k:11128 Zbl 1210.11090

[Green and Tao 2010] B. Green and T. Tao, "Linear equations in primes", Ann. of Math. (2) 171:3 (2010), 1753-1850. MR 2011j:11177 Zbl 1242.11071

[Iwaniec and Kowalski 2004] H. Iwaniec and E. Kowalski, Analytic number theory, American Mathematical Society Colloquium Publications 53, American Mathematical Society, Providence, RI, 2004. Zbl 1059.11001

[Kátai 1986] I. Kátai, "A remark on a theorem of H. Daboussi”, Acta Math. Hungar. 47:1-2 (1986), 223-225. MR 87j:11072 Zbl 0607.10034

[Kawada 1993] K. Kawada, "The prime $k$-tuplets in arithmetic progressions", Tsukuba J. Math. 17:1 (1993), 43-57. MR 94i:11068 Zbl 0797.11076

[Kawada 1995] K. Kawada, "A Montgomery-Hooley type theorem for prime $k$-tuplets", Acta Math. Hungar. 66:3 (1995), 177-200. MR 96h:11096 Zbl 0869.11069

[Matomäki and Radziwiłł 2015] K. Matomäki and M. Radziwiłł, "Multiplicative functions in short intervals", 2015. To appear in Ann. of Math. arXiv 1501.04585

[Mikawa 1992] H. Mikawa, "On prime twins in arithmetic progressions", Tsukuba J. Math. 16:2 (1992), 377-387. MR 94e:11101 Zbl 0778.11053

[Montgomery 1994] H. L. Montgomery, Ten lectures on the interface between analytic number theory and harmonic analysis, CBMS Regional Conference Series in Mathematics 84, American Mathematical Society, Providence, RI, 1994. MR 96i:11002 Zbl 0814.11001

[Montgomery and Vaughan 1977] H. L. Montgomery and R. C. Vaughan, "Exponential sums with multiplicative coefficients", Invent. Math. 43:1 (1977), 69-82. MR 56 \#15579 Zbl 0362.10036

[Montgomery and Vaughan 2007] H. L. Montgomery and R. C. Vaughan, Multiplicative number theory, I: Classical theory, Cambridge Studies in Advanced Mathematics 97, Cambridge Univ. Press, 2007. MR 2009b:11001 Zbl 1142.11001

Communicated by Andrew Granville

Received 2015-04-17 Revised 2015-08-28 Accepted 2015-10-06

ksmato@utu.fi

maksym.radziwill@gmail.com

tao@math.ucla.edu
Department of Mathematics and Statistics, University of Turku, Fl-20014 Turku, Finland

Department of Mathematics, Rutgers University, Hill Center for the Mathematical Sciences, 110 Frelinghuysen Rd., Piscataway, NJ 08854-8019, United States

Department of Mathematics, University of California Los Angeles, 405 Hilgard Avenue, Los Angeles, CA 90095-1555, United States 


\section{Algebra \& Number Theory}

msp.org/ant

\section{EDITORS}

MANAGING EDITOR

Bjorn Poonen

Massachusetts Institute of Technology

Cambridge, USA

\author{
EDITORIAL BOARD CHAIR \\ David Eisenbud \\ University of California \\ Berkeley, USA
}

BOARD OF EDITORS

Georgia Benkart

Dave Benson

Richard E. Borcherds

John H. Coates

J-L. Colliot-Thélène

Brian D. Conrad

Hélène Esnault

Hubert Flenner

Sergey Fomin

Edward Frenkel

Andrew Granville

Joseph Gubeladze

Roger Heath-Brown

Craig Huneke

Kiran S. Kedlaya

János Kollár

Yuri Manin

Philippe Michel
University of Wisconsin, Madison, USA

University of Aberdeen, Scotland

University of California, Berkeley, USA

University of Cambridge, UK

CNRS, Université Paris-Sud, France

Stanford University, USA

Freie Universität Berlin, Germany

Ruhr-Universität, Germany

University of Michigan, USA

University of California, Berkeley, USA

Université de Montréal, Canada

San Francisco State University, USA

Oxford University, UK

University of Virginia, USA

Univ. of California, San Diego, USA

Princeton University, USA

Northwestern University, USA

École Polytechnique Fédérale de Lausanne
Susan Montgomery

Shigefumi Mori

Raman Parimala

Jonathan Pila

Anand Pillay

Victor Reiner

Peter Sarnak

Joseph H. Silverman

Michael Singer

Vasudevan Srinivas

J. Toby Stafford

Ravi Vakil

Michel van den Bergh

Marie-France Vignéras

Kei-Ichi Watanabe

Efim Zelmanov

Shou-Wu Zhang
University of Southern California, USA

RIMS, Kyoto University, Japan

Emory University, USA

University of Oxford, UK

University of Notre Dame, USA

University of Minnesota, USA

Princeton University, USA

Brown University, USA

North Carolina State University, USA

Tata Inst. of Fund. Research, India

University of Michigan, USA

Stanford University, USA

Hasselt University, Belgium

Université Paris VII, France

Nihon University, Japan

University of California, San Diego, USA

Princeton University, USA

\section{PRODUCTION}

production@msp.org

Silvio Levy, Scientific Editor

See inside back cover or msp.org/ant for submission instructions.

The subscription price for 2015 is US $\$ 255 /$ year for the electronic version, and $\$ 440 /$ year ( $+\$ 55$, if shipping outside the US) for print and electronic. Subscriptions, requests for back issues and changes of subscribers address should be sent to MSP.

Algebra \& Number Theory (ISSN 1944-7833 electronic, 1937-0652 printed) at Mathematical Sciences Publishers, 798 Evans Hall \#3840, c/o University of California, Berkeley, CA 94720-3840 is published continuously online. Periodical rate postage paid at Berkeley, CA 94704, and additional mailing offices.

ANT peer review and production are managed by EditFLOW ${ }^{\circledR}$ from MSP.

\section{PUBLISHED BY}

- mathematical sciences publishers

nonprofit scientific publishing

http://msp.org/

(C) 2015 Mathematical Sciences Publishers 


\section{Algebra \& Number Theory}

Volume $9 \quad$ No. $9 \quad 2015$

Families of nearly ordinary Eisenstein series on unitary groups XIN WAN

Classifying orders in the Sklyanin algebra

Daniel Rogalski, Susan J. SierRa and J. Toby STAFFord

Chongying Dong, XingJun Lin and SiU-Hung NG

KAISA MATOMÄKI, MAKSYM RADZIWIŁŁ and TERENCE TAO 